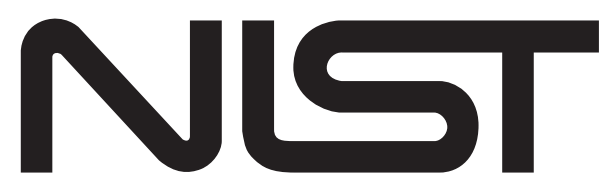

National Institute of

Standards and Technology

U.S. Department of Commerce

NIST Special Publication 250-90

\title{
Calibration Uncertainty for the NIST PM/AM Noise Standards
}

Archita Hati

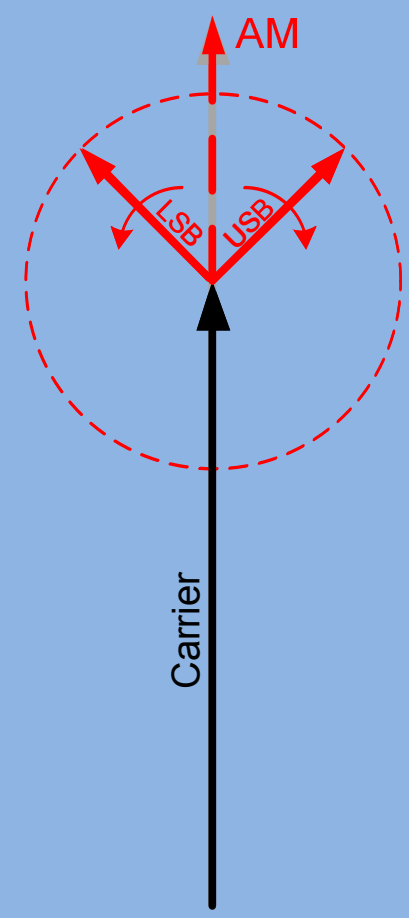

AM

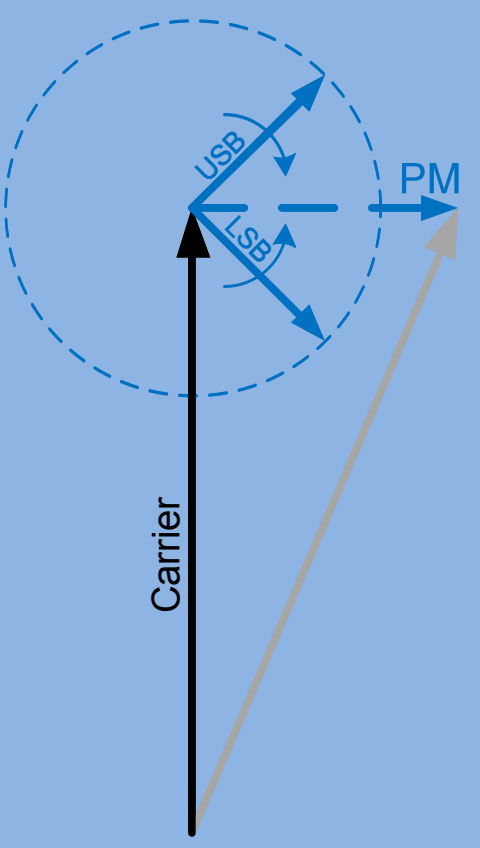

PM 
$\boldsymbol{T}$ he National Institute of Standards and Technology was established in 1988 by Congress to "assist industry in the development of technology ... needed to improve product quality, to modernize manufacturing processes, to ensure product reliability ... and to facilitate rapid commercialization ... of products based on new scientific discoveries."

NIST, originally founded as the National Bureau of Standards in 1901, works to strengthen U.S. industry's competitiveness; advance science and engineering; and improve public health, safety, and the environment. One of the agency's basic functions is to develop, maintain, and retain custody of the national standards of measurement, and provide the means and methods for comparing standards used in science, engineering, manufacturing, commerce, industry, and education with the standards adopted or recognized by the Federal Government.

As an agency of the U.S. Commerce Department's Technology Administration, NIST conducts basic and applied research in the physical sciences and engineering, and develops measurement techniques, test methods, standards, and related services. The Institute does generic and precompetitive work on new and advanced technologies. NIST's research facilities are located at Gaithersburg, MD 20899, and at Boulder, CO 80305. Major technical operating units and their principal activities are listed below. For more information visit the NIST Website at http://www.nist.gov, or contact the Publications and Program Inquiries Desk, 301-975-3058.

\section{Office of the Director}

- National Quality Program

-International and Academic Affairs

\section{Technology Services}

- Standards Services

- Technology Partnerships

- Measurement Services

- Information Services

-Weights and Measures

\section{Advanced Technology Program}

-Economic Assessment

-Information Technology and Applications

-Chemistry and Life Sciences

-Electronics and Photonics Technology

\section{Manufacturing Extension Partnership \\ Program}

-Regional Programs

- National Programs

·Program Development

\section{Electronics and Electrical Engineering}

Laboratory

-Microelectronics

- Law Enforcement Standards

-Electricity

-Semiconductor Electronics

-Radio-Frequency Technology ${ }^{1}$

-Electromagnetic Technology ${ }^{1}$

-Optoelectronics ${ }^{1}$

-Magnetic Technology ${ }^{1}$

\author{
Materials Science and Engineering \\ Laboratory \\ -Intelligent Processing of Materials \\ Ceramics \\ -Materials Reliability ${ }^{1}$ \\ -Polymers \\ -Metallurgy \\ -NIST Center for Neutron Research
}

\section{Chemical Science and Technology \\ Laboratory \\ -Biotechnology \\ -Process Measurements \\ -Surface and Microanalysis Science \\ -Physical and Chemical Properties ${ }^{2}$ \\ -Analytical Chemistry}

\author{
Physics Laboratory \\ -Electron and Optical Physics \\ -Atomic Physics \\ -Optical Technology \\ - Ionizing Radiation \\ -Time and Frequency ${ }^{1}$ \\ Quantum Physics ${ }^{1}$
}

\section{Manufacturing Engineering \\ Laboratory \\ $\cdot$ Precision Engineering \\ - Manufacturing Metrology \\ -Intelligent Systems \\ -Fabrication Technology \\ -Manufacturing Systems Integration}

\author{
Building and Fire Research Laboratory \\ -Applied Economics \\ -Materials and Construction Research \\ -Building Environment \\ -Fire Research
}

\author{
Information Technology Laboratory \\ - Mathematical and Computational Sciences ${ }^{2}$ \\ - Advanced Network Technologies \\ Computer Security \\ -Information Access \\ - Convergent Information Systems \\ -Information Services and Computing \\ -Software Diagnostics and Conformance Testing \\ -Statistical Engineering
}

\footnotetext{
${ }^{1}$ At Boulder, CO 80305

${ }^{2}$ Some elements at Boulder, $\mathrm{CO}$
} 


\section{Calibration Uncertainty for the NIST PMIAM Noise Standards}

Archita Hati

Craig Nelson

Neil Ashby

David Howe

Time and Frequency Division

Physical Measurement Laboratory

National Institute of Standards and Technology

325 Broadway

Boulder, Colorado 80305

July 2012

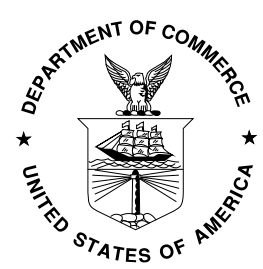

U.S. Department of Commerce Rebecca Blank, Acting Secretary

National Institute of Standards and Technology

Patrick Gallaher, Director 
Certain commercial entities, equipment, or materials may be identified in this document in order to describe an experimental procedure or concept adequately. Such identification is not intended to imply recommendation or endorsement by the National Institute of Standards and Technology, nor is it intended to imply that the entities, materials, or equipment are necessarily the best available for the purpose.

National Institute of Standards and Technology Special Publication 250-90 Natl. Inst. Stand. Technol. Spec. Publ. 250-90, 44 pages (July 2012) CODEN: NSPUE2

For sale by the Superintendent of Documents, U.S. Government Printing Office Internet: bookstore.gpo.gov — Phone: (202) 512-1800 — Fax: (202) 512-2250 Mail: Stop SSOP, Washington, DC 20402-0001 


\section{Contents}

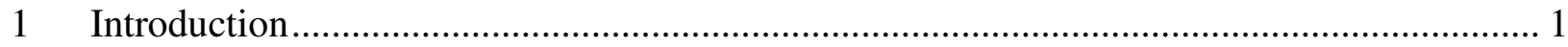

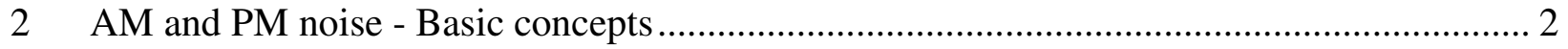

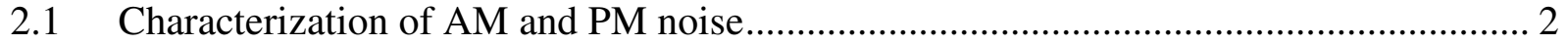

2.2 Overview of amplitude, phase and single sideband modulation ....................................... 3

2.2.1 Amplitude modulation ...................................................................................... 3

2.2.2 Phase modulation ...................................................................................... 3

2.2.3 Single-sideband modulation.............................................................................. 5

3 Description of the noise standard.............................................................................. 7

4 Measurement setup for calibration of the PM/AM noise standard ........................................ 9

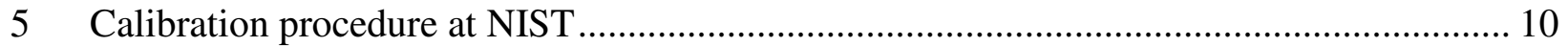

5.1 Measurement of the carrier power via down-conversion from the upper sideband....... 10

5.2 Measurement of the carrier power via down-conversion from the lower sideband....... 10

5.3 Measurement of the noise power spectral density down-converted from both the upper and the lower sidebands ................................................................................. 11

5.4 Measurement of the noise floor power spectral density ............................................. 12

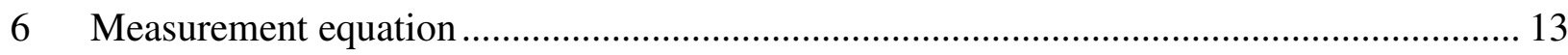

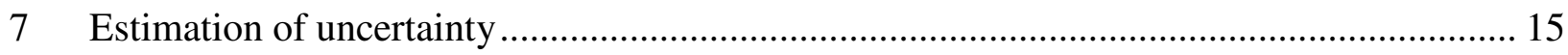

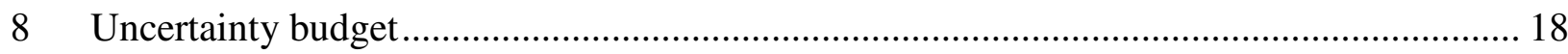

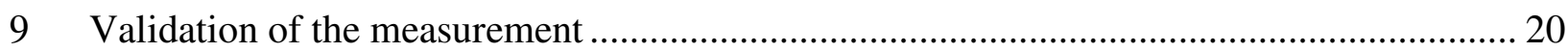

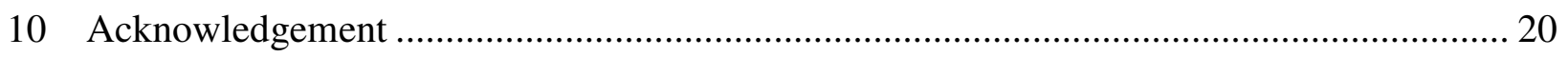

Appendix A Verification of the baseband measurement equation .............................................21

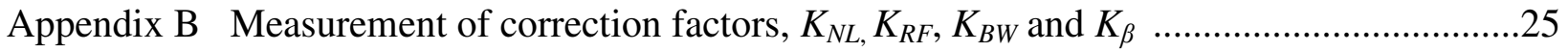

Appendix C Error of signal power measurements in the presence of background noise ............28

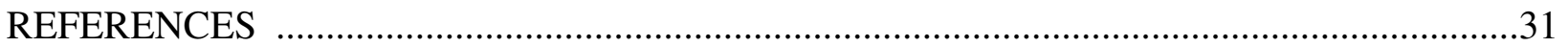




\section{Notations}

$\begin{array}{lll}\text { Acronym } & \text { Meaning } \\ \text { AM } & & \text { Amplitude Modulation } \\ \text { BPF } & & \text { Bandpass Filter } \\ \text { BW } & \text { Bandwidth } \\ \text { dB } & \text { Decibel } \\ \text { dBm } & \text { Decibel relative to 1 milliwatt } \\ \text { DDPNMS } & \text { Direct Digital Phase Noise Measurement System } \\ \text { FFT } & \text { Fast Fourier Transform } \\ \text { IF } & \text { Intermediate Frequency } \\ \text { LO } & \text { Local Oscillator } \\ \text { LPF } & \text { Low-Pass Filter } \\ \text { LSB } & \text { Lower Sideband } \\ \text { PDLMS } & \text { Photonic Delay Line Measurement System } \\ \text { PM } & \text { Phase Modulation } \\ \text { PSD } & \text { Power Spectral Density } \\ \text { rf } & \text { Radio Frequency } \\ \text { RF } & \text { Reference Frequency } \\ \text { SNR } & \text { Signal-to-Noise Power Ratio } \\ \text { SSB } & \text { Single Sideband } \\ \text { USB } & \text { Upper Sideband } \\ \text { VSWR } & \text { Voltage Standing-Wave Ratio }\end{array}$

\section{Symbol Meaning}

$\alpha(t) \quad$ Instantaneous amplitude fluctuations

$\beta \quad$ Phase modulation index

$\delta_{N L} \quad$ Maximum error of $K_{N L}$ from unity

$\delta_{R F} \quad$ Maximum error of $K_{R F}$ from unity

$\mathcal{E}_{\beta} \quad$ Correction factor due to the small angle modulation approximation

$\phi(t) \quad$ Instantaneous phase fluctuations

$\Delta \phi_{\text {peak }} \quad$ Peak phase deviation

$\Delta \phi_{r m s} \quad$ Root-mean-square phase deviation

$\gamma \quad$ Noise power in $1 \mathrm{~Hz}$ bandwidth

$v_{0} \quad$ Carrier frequency

$v_{\mathrm{LO}} \quad$ Carrier frequency at the LO port of the mixer

$v_{\mathrm{RF}} \quad$ Carrier frequency at the RF port of the mixer

$\sigma_{\beta} \quad$ Fractional uncertainty due to the small angle modulation approximation

$\sigma_{\text {Beat }} \quad$ Fractional uncertainty in the lower-sideband beat measurement

$\sigma_{\text {Beat }} \quad$ Fractional uncertainty in the upper-sideband beat measurement

$\sigma_{B \text {-FFTAve }} \quad$ Uncertainty due to the number of FFT averages for beat measurement

$\sigma_{B W} \quad$ Fractional uncertainty in the estimated measurement bandwidth for the power 
spectral density function

$\sigma_{C} \quad$ Combined fractional uncertainty

$\sigma_{L R} \quad$ Uncertainty due the long-term reproducibility of the PM/AM noise standard between calibration cycles

$\sigma_{N-F F T A v e} \quad$ Uncertainty due to the number of FFT averages for noise measurement

$\sigma_{N L}$

$\sigma_{\text {NoiseOn }}$

$\sigma_{R F}$

$\sigma_{S R}$

$\omega_{0}$

$\omega_{m}$

$\mathrm{BW}_{\text {Actual }}$

$\mathrm{BW}_{\mathrm{Est}}$

$f$

$f_{\mathrm{L}}$

$f_{\mathrm{U}}$

$g_{I F}$

$g_{R F}$

$g_{V S W R}$

$g_{L V L}$

$G$

$J_{n}$

$k$

$K_{\beta}$

$K_{B W}$

$K_{N L}$

$K_{R F}$

$\mathscr{L}(f)$

m

$n_{\text {set }}$

$N_{\text {Beat }}$

$N_{\text {Noise }}$

$O$

$P_{c}$

$P_{\text {carrier }}$

$P_{\text {C-Beat }}$

$P_{\text {noise }}$

$P_{N-B e a t}$

$P_{S S B-A M}$

$P_{S S B-P M}$

$S_{\alpha}(f)$

$S_{\phi}(f)$

Fractional uncertainty due to the FFT analyzer and mixer nonlinearity at baseband

Fractional uncertainty in the noise measurement with the noise source on

Fractional uncertainty due to the frequency response of the down-convertor (includes offset signal generator and mixer) at $\mathrm{rf}$

Uncertainty due to the short-term repeatability between measurements

Angular frequency of the carrier signal

Angular modulation frequency

Actual bandwidth

Estimated bandwidth

Fourier or offset frequency

Lower frequency limit of the integrated phase noise

Upper frequency limit of the integrated phase noise

Gain of the down-converter at the baseband

Gain of the down-converter at its two operating frequencies, $v_{\mathrm{RF}}$ and $v_{\mathrm{LO}}$

Gain of the down-converter due to VSWR mismatch

Gain of the down-converter for a specific power level at the RF port

Gain of the down-converter

$n$-th order Bessel function

Coverage factor

Correction factor applied to $\mathscr{L}(f)$ due to the small angle modulation approximation

Correction factor for incorrect estimation of the actual measurement bandwidth for the power spectral density function

Correction factor due to the FFT analyzer and mixer nonlinearity at baseband

Correction factor due to the frequency response of the down-convertor (includes offset signal generator and mixer) at radio frequencies

Single sideband phase noise equal to one half of $S_{\phi}(f)$

Amplitude modulation index

Number of repeated measurement sets in a given calibration

Number of FFT averages for beat measurement

Number of FFT averages for noise measurement

Higher-order terms of Taylor expansion

Carrier power

Power level at the RF port of the mixer during carrier power measurement

Carrier power after down-conversion to baseband

Power level at the RF port of the mixer during the noise measurement

Noise power after down-conversion to baseband

Single-sideband AM signal power

Single-sideband PM signal power

One-sided double-sideband power spectral density of amplitude fluctuations

One-sided double-sideband power spectral density of random phase fluctuations 


\begin{tabular}{|c|c|}
\hline$u_{C}$ & Combined uncorrelated uncertainty \\
\hline$U$ & Expanded uncertainty \\
\hline $\mathrm{v}(t)$ & Instantaneous voltage fluctuations \\
\hline$V_{0}$ & Peak voltage of the carrier signal \\
\hline$V_{\text {Baseband }}$ & $\begin{array}{l}\text { Folded double-sideband down-converted voltage of the PM/AM noise standard at } \\
\text { baseband }\end{array}$ \\
\hline$V_{\text {Beat- }}$ & Voltage of the lower-sideband beat frequency \\
\hline$V_{\text {Beat+ }}$ & Voltage of the upper-sideband beat frequency \\
\hline$V_{\text {BeatAve }}$ & Average voltage of the upper- and lower-sideband beats \\
\hline$V_{\text {Beat }-, M}$ & Measured voltage of the lower-sideband beat \\
\hline$V_{\text {Beat+, } M}$ & Measured voltage of the upper-sideband beat \\
\hline$V_{\text {NoiseOff }}$ & Voltage of the additive noise at baseband with noise source off \\
\hline$V_{\text {NoiseOn }}$ & Voltage of the additive noise at baseband with noise source on \\
\hline$V_{\text {NoiseOff, } M}$ & Measured voltage of the additive noise at baseband with noise source off \\
\hline$V_{\text {NoiseOn }}, M$ & Measured voltage of the additive noise at baseband with noise source on \\
\hline$V_{P N S T}$ & Signal voltage of the PM/AM noise standard at $\mathrm{rf}$ \\
\hline$V_{P N S T(\text { CarrierOn })}$ & $\begin{array}{l}\text { Signal voltage of the noise standard when the additive noise is off and the carrier } \\
\text { is enabled }\end{array}$ \\
\hline$V_{P N S T(N o i s e O n)}$ & $\begin{array}{l}\text { Signal voltage of the noise standard at baseband when the additive noise is } \\
\text { enabled and the carrier is off }\end{array}$ \\
\hline$V_{S S B}$ & Peak voltage of the single-sideband tone \\
\hline
\end{tabular}




\section{Introduction}

In this document, we provide the total (or combined) uncertainty of phase modulation (PM) and amplitude modulation (AM) noise measurement of the NIST portable noise standard calculated from the individual measurement uncertainties. NIST provides an on-site calibration service (77135C) of the accuracy of PM and AM noise and noise floor of the measurement systems at the customer's site by use of two separate portable PM/AM secondary noise standards [1]. One noise standard is for carrier frequencies of $5 \mathrm{MHz}, 10 \mathrm{MHz}$, and $100 \mathrm{MHz}$, designated as model 510100 [2], [3], and the other for carrier frequencies of $10.6 \mathrm{GHz}, 21.2 \mathrm{GHz}$, and $42.4 \mathrm{GHz}$, designated as model 102040 [4].

The PM and AM noise of the noise standard are usually measured twice at NIST, once before sending it to the customer (the "out" measurement) and a second time after receiving it back (the "in" measurement). The customer uses its PM/AM noise measurement system and measures the calibrated noise level of the NIST secondary noise standard. Customer results are compared with the average of the "in" and "out" measurements at NIST. If the results are within the measurement uncertainty then the calibration is certified and a "PASS" calibration report is issued. When there is significant discrepancy in the results, the customer is advised to recheck the measurement configuration and re-measure. If the customer results cannot be achieved within the measurement uncertainty, a "FAIL" calibration report is then issued.

The purpose of this document is to describe the calibration uncertainty for the NIST PM/AM noise standard. The document is organized as follows. In Section 2, we first introduce the basic concepts of AM and PM noise. We briefly discuss the characterization of AM and PM noise in Sub-section 2.1, and an overview of different types of signal modulation is presented in Subsection 2.2. Section 3 describes the working principle of the PM/AM noise standard, and in Section 4 the experimental set-up for calibrating the standard is presented. In Section 5 a detailed step-by-step calibration procedure is provided. The measurement equation, a detailed uncertainty calculation and the uncertainty budget for the noise standard are presented in Sections 6, 7 and 8, respectively. Finally, in Section 9, a brief discussion for validating the calibration results is presented. This document is supported by three appendices. Appendix A provides verification that the baseband measurement equation is equal to the original definition of single sideband (SSB) PM noise at the radio frequency (rf). Measurement of different correction factors involved in the calibration of the noise standard is described in Appendix B. Finally, Appendix C presents a calculation for the error of a signal power measurement in the presence of background noise 


\section{AM and PM noise - Basic concepts}

In this section we briefly describe the basic concept of phase-modulated (PM) noise and amplitude-modulated (AM) noise and their characterization in the frequency domain [5-8]. We also discuss the basic theory behind the PM/AM noise standard.

\subsection{Characterization of AM and PM noise}

In the real world, an oscillator outputs a signal that fluctuates in both amplitude and phase. This signal can be mathematically represented by

$$
\mathrm{v}(t)=V_{0}[1+\alpha(t)] \cos \left[\omega_{0} t+\phi(t)\right]
$$

where $V_{0}=\sqrt{2} V_{r m s}$ is the peak amplitude, $\alpha(t)$ is the normalized instantaneous amplitude fluctuation, $\omega_{0}=2 \pi v_{0}$ is the angular frequency of the carrier, $\phi(t)$ is the instantaneous phase fluctuation, $V_{r m s}$ is the root-mean-square $(\mathrm{rms})$ voltage, and $v_{0}$ is the carrier frequency.

In the frequency domain, the amplitude stability of a signal is characterized by the power spectral density (PSD) of instantaneous amplitude fluctuations $S_{\alpha}(f)$, given by

$$
S_{\alpha}(f)=\frac{\langle\alpha(f)\rangle^{2}}{B W},
$$

where $\langle\alpha(f)\rangle^{2}$ is the mean-square normalized amplitude fluctuation at an offset or Fourier frequency $f(0<f<\infty)$ from the carrier and BW is the bandwidth of the measurement system. The unit of $S_{\alpha}(f)$ is $1 / \mathrm{Hz}$. Similarly, in the frequency domain, the phase instability of a signal is characterized by the PSD of instantaneous phase fluctuations $S_{\phi}(f)$, given by

$$
S_{\phi}(f)=\frac{\left\langle\Delta \phi_{r m s}(f)\right\rangle^{2}}{B W},
$$

where $\left\langle\Delta \phi_{r m s}(f)\right\rangle^{2}$ is the mean-square phase fluctuation at an offset frequency $f(0<f<\infty)$ from the carrier frequency $v_{0}$. The unit of $S_{\phi}(f)$ is $\operatorname{rad}^{2} / \mathrm{Hz}$. Since $f$ ranges from zero to infinity and since both $S_{\alpha}(f)$ and $S_{\phi}(f)$ include the fluctuations from upper and lower sidebands of the carrier, these two quantities are single-sided double-sideband units of measure.

The PM noise unit of measure recommended by the IEEE [5] is $\mathscr{L}(f)$, defined as

$$
\mathscr{L}(f) \equiv \frac{S_{\phi}(f)}{2}
$$


$\mathscr{L}(f)$ includes the fluctuations from only one sideband of the carrier, and hence it is a singlesideband unit of measure. When $\mathscr{L}(f)$ is expressed in the form $10 \log [\mathscr{L}(f)]$, its unit is $\mathrm{dBc} / \mathrm{Hz}$, that is $\mathrm{dB}$ below the carrier in a $1 \mathrm{~Hz}$ bandwidth. The logarithm is computed to the base 10 .

Also, when the integrated PM noise for offset frequencies $0<f<\infty$ is less than $0.01 \operatorname{rad}^{2}, \mathscr{L}(f)$ can be viewed as the ratio of phase noise power per unit bandwidth in a single sideband to power in the carrier [See later Eq.(12)].

\subsection{Overview of amplitude, phase and single sideband modulation}

Before we discuss the working principle of the NIST PM/AM noise standard, it is worthwhile to briefly discuss different types of signal modulation.

\subsubsection{Amplitude modulation}

In amplitude modulation, the carrier amplitude varies with the modulating baseband signal while the frequency and phase are not modulated. The simplest AM signal is produced when the modulating signal is a sinusoid at frequency $\omega_{m}$ [9], [10]. The resultant modulated signal also called single-tone AM, is given by

$$
\begin{aligned}
\mathrm{v}_{A M}(t) & =V_{0}\left[1+m \cos \omega_{m} t\right] \cos \omega_{0} t \\
& =V_{0} \operatorname{Re}\left\{e^{i \omega_{0} t}\left[1+\frac{m}{2}\left(e^{i \omega_{m} t}+e^{-i \omega_{m} t}\right)\right]\right\},
\end{aligned}
$$

where $m$ is the amplitude modulation index. The two sidebands are symmetrical about the carrier frequency and are equal in magnitude. The ratio of SSB AM power $\left(P_{S S B-A M}\right)$ to carrier power $\left(P_{c}\right)$ is

$$
\frac{P_{S S B-A M}}{P_{C}}=\frac{m^{2}}{4} .
$$

For AM, the resultant vector sum of the sidebands is always in phase with the carrier (Figure 1).

\subsubsection{Phase modulation}

In the case of phase modulation, the modulating signal leaves the amplitude of the carrier unchanged. Instead, the phase of the carrier varies with the modulating signal [9], [10]. A singletone PM signal can be represented by

$$
\begin{aligned}
\mathrm{v}(t) & =V_{0} \cos \left[\omega_{0} t+\beta \sin \omega_{m} t\right] \\
& =V_{0} \operatorname{Re}\left\{e^{i \omega_{0} t} e^{i \beta \sin \omega_{m} t}\right\},
\end{aligned}
$$


where $\beta$ is the phase-modulation index (or peak phase deviation, also denoted by $\Delta \phi_{\text {peak }}$ ). The second exponential term in the curly bracket is a periodic signal with period $2 \pi / \omega_{m}$ and can be expanded by the exponential Fourier series

$$
e^{i \beta \sin \omega_{m} t}=\sum_{n=-\infty}^{\infty} J_{n}(\beta) e^{i n \omega_{m} t}
$$

$J_{n}(\beta)$ is the $n^{\text {th }}$ - order Bessel function of the first kind [11]. For small modulation index $(\beta<<$ $1)$,

$$
\begin{aligned}
& J_{0}(\beta)=1 \\
& J_{1}(\beta)=\beta / 2 \\
& J_{n}(\beta)=0 \quad|n|>1 \text {. }
\end{aligned}
$$

Also, from symmetry, $J_{-n}(\beta)=(-1)^{n} J_{n}(\beta)$; therefore, Eq. (7) becomes

$$
\mathrm{v}(t)=V_{0} \operatorname{Re}\left\{e^{i \omega_{0} t}\left[1+\frac{\beta}{2}\left(e^{i \omega_{m} t}-e^{-i \omega_{m} t}\right)\right]\right\}
$$

This resembles the AM case in Eq. (5), except that in PM, the phase of the lower sideband is reversed, and the resultant vector sum of the sidebands is always in phase quadrature with the carrier (Figure 1).

Further, the ratio of SSB PM power $\left(P_{S S B-P M}\right)$ to carrier power is

$$
\frac{P_{S S B-P M}}{P_{C}}=\frac{\beta^{2}}{4}=\frac{\Delta \phi_{\text {peak }}^{2}}{4} .
$$

As mentioned in Section 2.1, when the integrated PM noise for offset frequencies $0<f<\infty$ is less than $0.01 \mathrm{rad}^{2}, \mathscr{L}(f)$ can be viewed as the ratio of phase-noise power in a single sideband to the carrier power. This can also be shown analytically by use of Eqs. (3), (4) and (11), as follows:

$$
\mathscr{L}(f)=\frac{1}{2} S_{\phi}(f)=\frac{\left\langle\Delta \phi_{r m s}(f)\right\rangle^{2}}{2 B W}=\frac{\left\langle\Delta \phi_{\text {peak }}(f)\right\rangle^{2}}{4 B W}=\left.\left\langle\frac{P_{S S B-P M}(f)}{P_{C}}\right\rangle\right|_{B W=1 \mathrm{~Hz}},
$$

when 


$$
\sqrt{2 \int_{0}^{\infty} \mathrm{S}_{\phi}(f) \mathrm{d} f} \quad<0.1 \quad \operatorname{rad}_{\text {peak }} .
$$

However, the practical values for the lower and upper integration limits, $f_{L}$ and $f_{U}$, are respectively the reciprocal of the measurement time and system half-bandwidth.

The condition of Eq. (13) follows by defining a higher order correction, $\varepsilon_{\beta}$, as a normalized departure of Eq. (11) from the small angle modulation approximation given by

$$
\varepsilon_{\beta}=\left(\frac{J_{1}(\beta) / J_{0}(\beta)}{\beta / 2}\right)^{2} .
$$

Then the error $\left(\varepsilon_{\beta}-1\right)$ in $\mathscr{L}(f)$ for $\beta<<0.1$ is $0.3 \%(0.01 \mathrm{~dB})$ or less.

\subsubsection{Single-sideband modulation}

In this Section, we discuss briefly single-sideband modulation, as this is necessary to explain the working principle of the PM/AM noise standard. A single-tone upper SSB signal is represented by

$$
\begin{aligned}
\mathrm{v}(t) & =V_{0} \cos \omega_{0} t+V_{S S B} \cos \left(\omega_{0}+\omega_{m}\right) t \\
& =V_{0} \operatorname{Re}\left[e^{i \omega_{0} t}\left(1+\frac{V_{S S B}}{V_{0}} e^{i \omega_{m} t}\right)\right] \\
& =V_{0} \operatorname{Re}\left\{e^{i \omega_{0} t}[1+\underbrace{\frac{V_{S S B}}{2 V_{0}}\left(e^{i \omega_{m} t}+e^{-i \omega_{m} t}\right)}_{A M}+\underbrace{\frac{V_{S S B}}{2 V_{0}}\left(e^{i \omega_{m} t}-e^{-i \omega_{m} t}\right)}_{P M}]\right\},
\end{aligned}
$$

where $V_{S S B}$ is the peak voltage of the single-sideband tone at $\left(\omega_{0}+\omega_{m}\right)$. Now comparing Eq. (15) with (5) and (10), the second term within the square bracket represents AM, and the last term represents PM. Therefore, the SSB signal can be envisioned as a combination of four signals of equal amplitude; two of them consist of pure AM, and the other two consist of pure PM. For a SSB signal, half of the power is present in AM and other half is present in PM. Eq. (15) can also be explained in other words as follows: the two lower sideband terms $\left(e^{-i \omega_{m} t}\right)$ are $180^{\circ}$ out of phase, and thus cancel, and the upper sideband terms $\left(e^{i \omega_{n} t}\right)$ are in phase, and thus add coherently, resulting in a SSB modulation.

Now if instead of a single tone, there is additive white noise, the theory of single-tone SSB modulation can still be applied [12], [13]. Consider an upper noise sideband at " $v_{0}+f$ " with associated noise power $\gamma$ in $1 \mathrm{~Hz}$ bandwidth. It will produce upper and lower PM and AM noise 
sidebands, each having average power $\gamma^{2} / 4$. If there is a similar amount of uncorrelated white noise power in the lower sideband at " $v_{0^{-}} f$ ", it also will produce four signals, two AM and two PM of average power $\gamma^{2} / 4$. The contribution from the lower and upper sidebands produce on average equal amounts of PM and AM noise; however, the phase difference between them varies randomly with time.

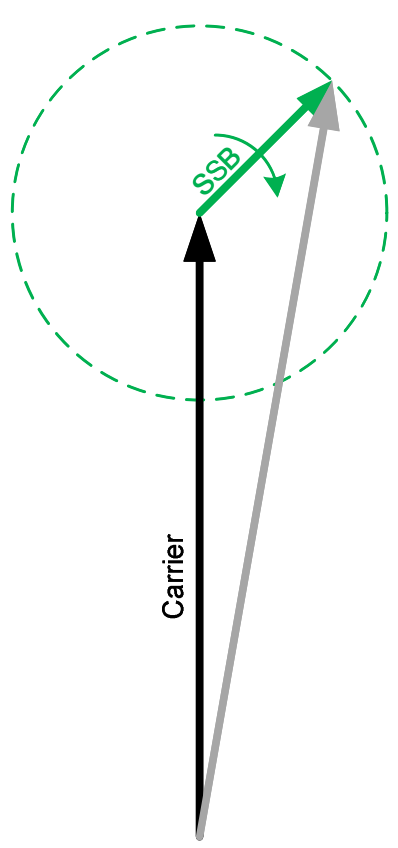

SSB

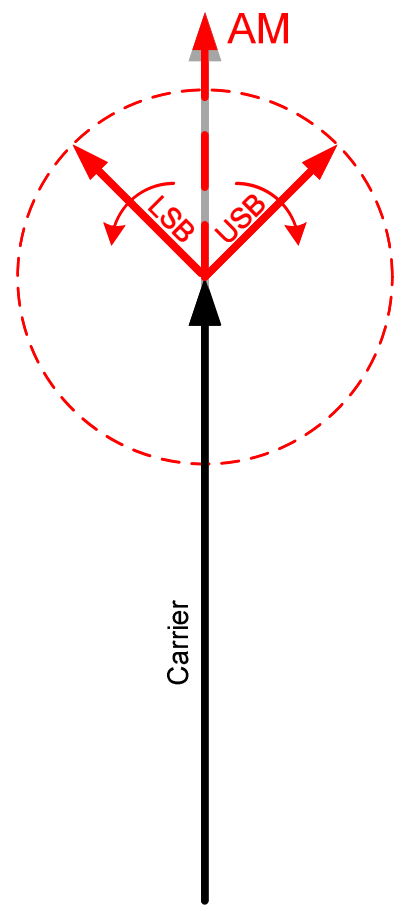

AM

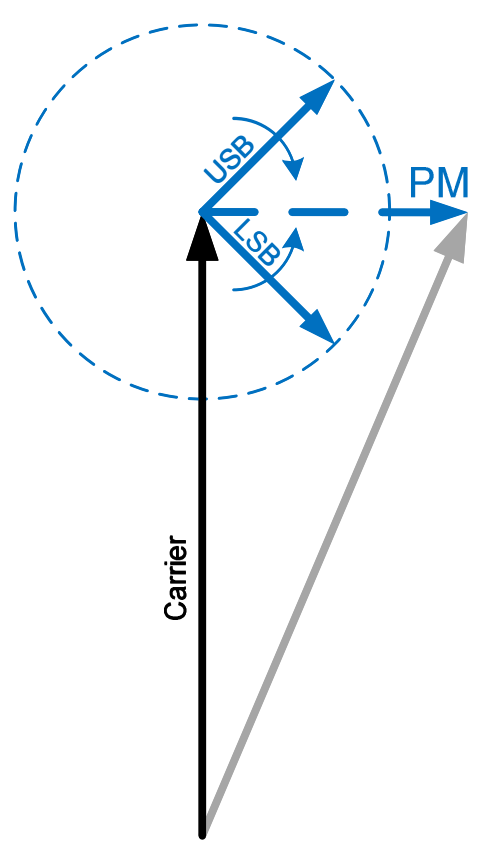

PM

Figure 1. Phasor diagrams of the amplitude, phase and SSB modulation. LSB is the lower sideband and USB is the upper sideband. The gray vector indicates the resultant of the carrier and sidebands. 


\section{Description of the noise standard}

The block diagram of the noise standard at $5 \mathrm{MHz}, 10 \mathrm{MHz}$, and $100 \mathrm{MHz}$ is shown in Figure 2 . The detailed description of this noise standard can be found in [2]. The noise standard has two outputs, one clean unmodulated reference and another modulated. The powers of the two output signals are approximately $+15 \mathrm{dBm}$ for each carrier frequency.

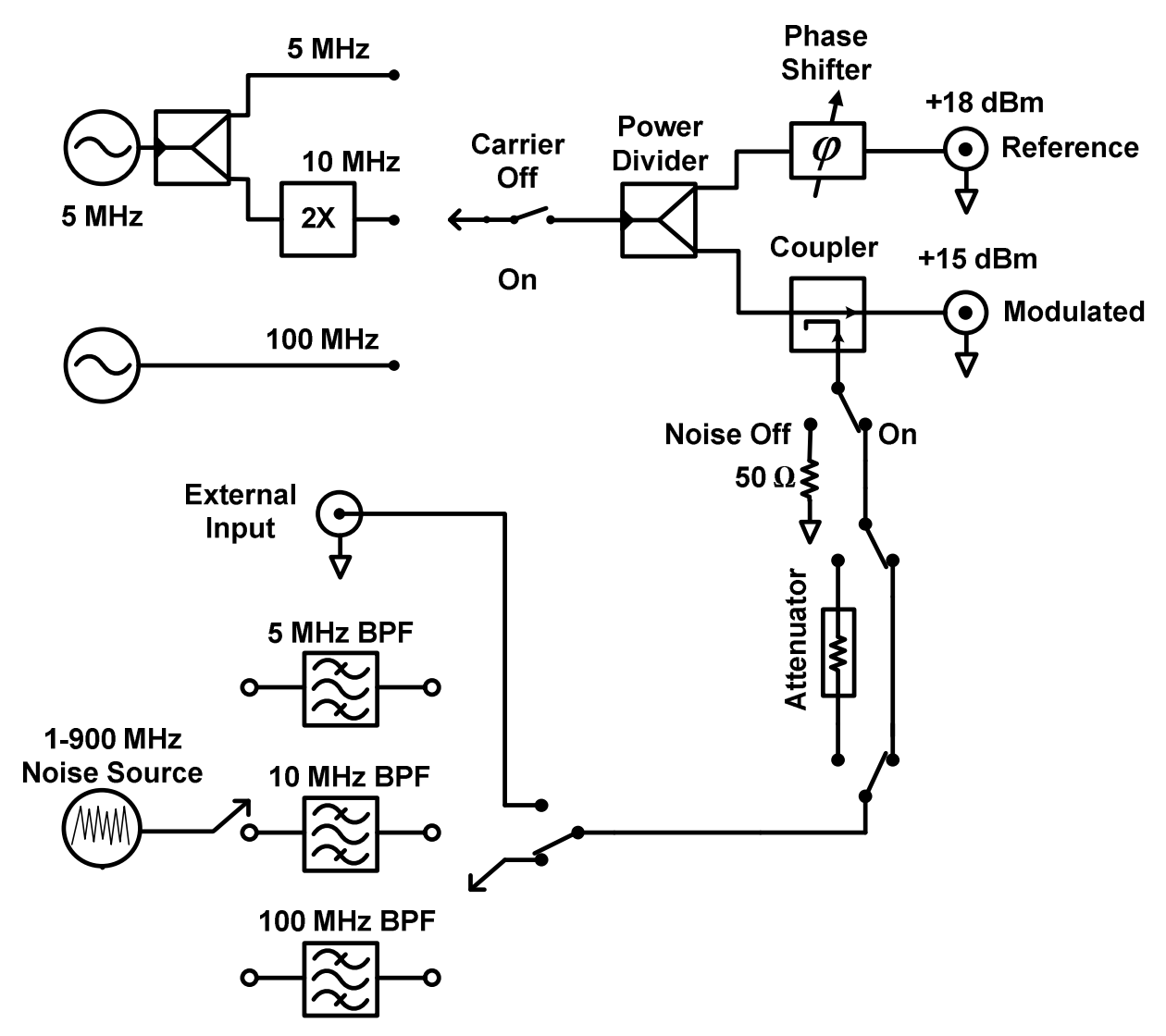

Figure 2. Block diagram of 510100 PM/AM noise standard, which selects one of three frequencies of $5 \mathrm{MHz}, 10 \mathrm{MHz}$, and $100 \mathrm{MHz}$. BPF is the bandpass filter

A calibrated level of bandpass-limited Gaussian noise is added to the "Modulated Output" of the standard to create equal levels of PM and AM noise. The level of added noise is selected such that it satisfies Eq. (13), and is at least $40 \mathrm{~dB}$ above the noise floor of most measurement systems. This calibrated noise level is typically constant in magnitude to better than $\pm 0.5 \mathrm{~dB}$ for Fourier frequencies from dc to $10 \%$ of the carrier frequency. For the $5 \mathrm{MHz}$ standard, the calibrated noise level is approximately $-110 \mathrm{dBc} / \mathrm{Hz}$ with a $3.5 \mathrm{MHz}$ bandwidth. The residual $\mathrm{PM}$ and AM noise between these two outputs is exceptionally low [2]. Typically, the differential PM noise between the two output signals is less than $-190 \mathrm{dBc} / \mathrm{Hz}$ for Fourier offsets of $10 \mathrm{kHz}$ and above. 
The modulated output has precisely equal PM and AM noise, since there is no phase coherence between the signal and the noise, as described in 2.2.3 and [12]. The power spectral density (PSD) of the SSB PM noise, $[\mathscr{L}(f)]$, and AM noise, $\left[1 / 2 \mathrm{~S}_{\alpha}(f)\right]$, generated by the noise standard is given by

$$
\mathscr{L}(f)=\frac{1}{2} S_{\alpha}(f)=\frac{P S D\left[\text { Noise }\left(v_{0}-f\right)\right]+P S D\left[\text { Noise }\left(v_{0}+f\right)\right]}{4 \text { Carrier Power }},
$$

when Eq. (13) is satisfied [2]. One factor of two in the denominator arises because half of the additive noise power appears as PM and half as AM. The other factor of 2 is due to the equal distribution of noise power between the upper and lower sidebands.

$\mathscr{L}(f)$ is constant with frequency from dc to approximately the half-bandwidth of the filter, assuming that the added noise is constant in amplitude from $v_{0}-f$ to $v_{0}+f$. 


\section{Measurement setup for calibration of the PM/AM noise standard}

To measure $\mathscr{L}(f)$ at multiple offset frequencies, $f$, a digital fast Fourier transform (FFT) spectrum analyzer is used to take advantage of its speed, high dynamic range and flexibility in measurement bandwidth. To measure beyond the upper frequency capability of the FFT, a heterodyne down-converter is used to convert the output of the PM/AM noise standard to an intermediate frequency (IF) that can be conveniently measured by the FFT analyzer.

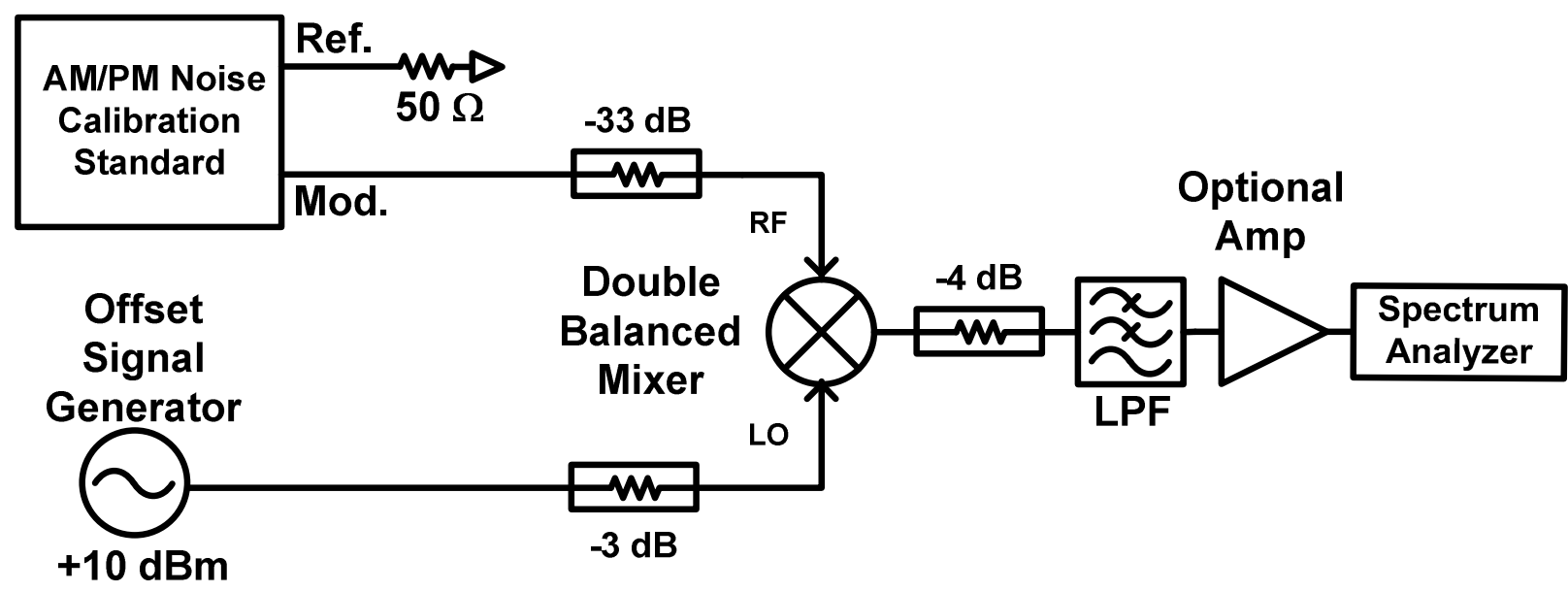

Figure 3. Block diagram of a heterodyne measurement system used to calibrate the 510100 PM/AM noise standard

The block diagram of the heterodyne down-converter used to calibrate a PM/AM noise standard at NIST is shown in Figure 3. It is composed of a double-balanced mixer (LO level $+7 \mathrm{dBm}$ ), an offset signal generator, and a low-pass filter (LPF). The modulated port of the noise standard is connected to the reference frequency (RF) mixer port via a large attenuator. The large attenuator at the RF port ensures that the signal from the PM/AM noise standard is far below the saturation point of the mixer and that it is operating as a linear frequency down-converter. The small attenuators on the local oscillator (LO) and IF ports are used to stabilize the impedance of the mixer. A LPF is used after the mixer to reject the sum of the two mixed frequencies and pass the difference signal. 


\section{Calibration procedure at NIST}

The offset generator and the PM/AM noise standard are turned on and allowed to stabilize for at least 24 hours. The offset signal generator is connected to the LO port of the down-converter and adjusted to $+10 \mathrm{dBm}$. If possible, the offset generator and the PM/AM standard are also locked to a common frequency reference. The reference port of the PM/AM noise standard is terminated with $50 \Omega$, and the modulated output port is connected to the highly attenuated RF port of the down-converter. The noise and the carrier of the PM/AM noise standard can be independently enabled. This allows the carrier and the noise to be independently characterized, utilizing the full dynamic range of the FFT device.

A typical calibration consists of a set of individual calibration points at different offset frequencies from the carrier. This set of offset frequencies is typically logarithmically spaced, 3 to 5 points per decade, spanning from $1 \mathrm{~Hz}$ to $10 \mathrm{MHz}$ or a maximum of $10 \%$ of the carrier frequency. For each offset frequency, $f$, in the calibration set, four measurements are made and used to calculate the phase noise generated at that offset frequency. These four measurement steps are graphically shown in Figure 4 and described in the following four sub-sections.

\subsection{Measurement of the carrier power via down-conversion from the upper sideband}

The carrier power of the PM/AM standard is turned $\mathrm{ON}$ and the noise is turned OFF. The frequency of the offset generator is set to $v_{0}+f$. The carrier at $v_{0}$ from the PM/AM standard and the signal from the offset generator at $v_{0}+f$ are mixed in the down-converter creating a baseband beat at frequency $f$. The power in the baseband signal at $f$ will be proportional to the carrier power of the PM/AM noise standard at $v_{0}$. The FFT analyzer range is set to auto to select the maximum dynamic range, and a measurement of the signal power at $f$ is made and recorded in units of $V_{r m s}^{2}$, utilizing a Flattop window. Also a measurement of the signal-to-noise power ratio (SNR) between the beat signal and the background noise is recorded. This measurement is graphically shown in Figure 4(a).

\subsection{Measurement of the carrier power via down-conversion from the lower sideband}

Similar to the previous step, the carrier power of the PM/AM standard stays ON and the noise is OFF. The frequency of the offset generator is set to $v_{0}-f$. The carrier at $v_{0}$ from the PM/AM standard and the signal from the offset generator at $v_{0}-f$ are mixed in the down-converter, creating a baseband beat at frequency $f$. The power in the baseband signal at $f$ will be proportional to the carrier power of the PM/AM noise standard at $v_{0}$. The FFT analyzer is autoranged for maximum dynamic range, and a measurement of the signal power at $f$ is made and recorded in units of $V_{r m s}^{2}$ by use of a Flattop window. Also, a measurement of the signal-tonoise ratio (SNR) between the beat signal and the background noise is recorded. This measurement is graphically shown in Figure 4(b). 


\subsection{Measurement of the noise power spectral density down-converted from both the upper and the lower sidebands}

The carrier power of the PM/AM standard is then turned OFF and the noise is turned ON. The frequency of the offset generator is set to $v_{0}$. The double-sideband noise spectrum about $v_{0}$ is down-converted to baseband by mixing with the offset generator at $v_{0}$. The PSD of the baseband noise at $f$ is proportional to the PSD of the double-sideband noise of the PM/AM noise standard about $v_{0}$. The FFT analyzer is auto-ranged for maximum dynamic range, and a measurement of the down-converted PSD at $f$ is made and recorded in units of $V_{r m s}^{2} / H z$, by use of a low-leakage Hanning window [14]. This measurement is graphically shown in Figure 4(c).

RF Signal Baseband

(a)

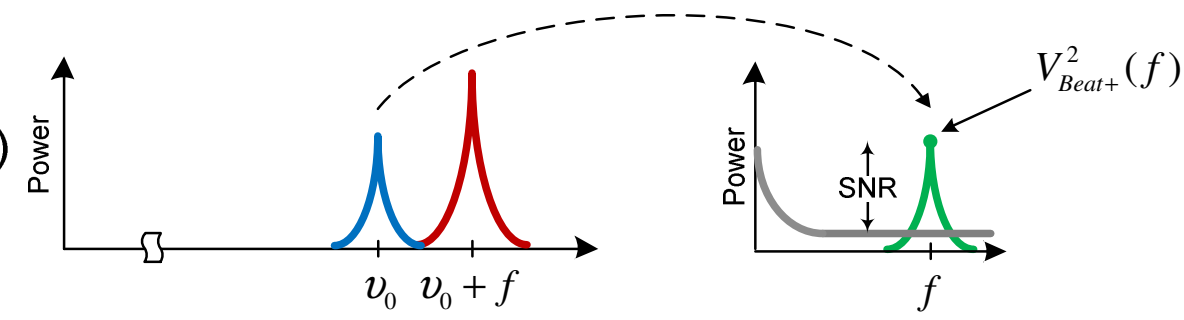

(b)

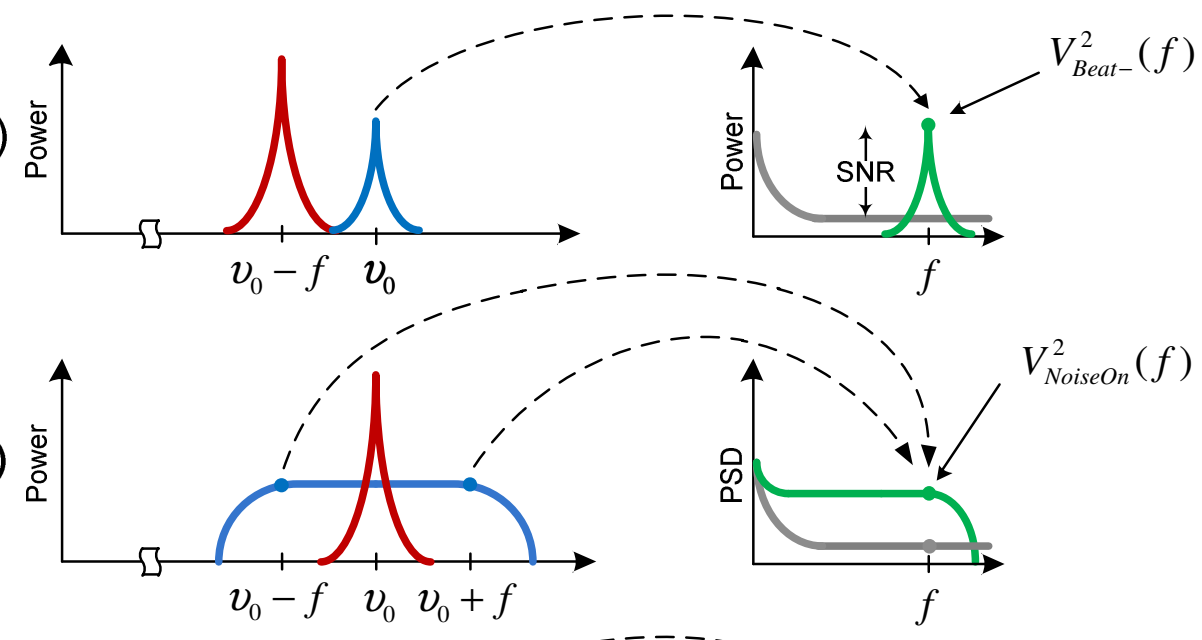

(c)

(d)

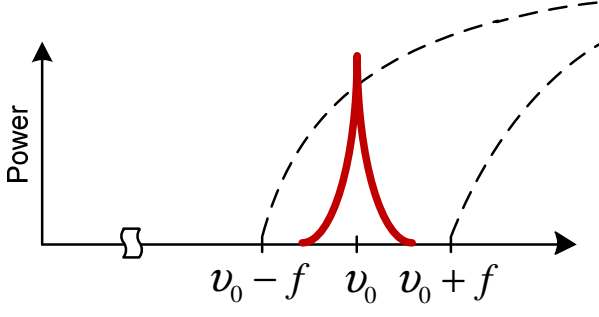

Frequency $[\mathrm{Hz}]$

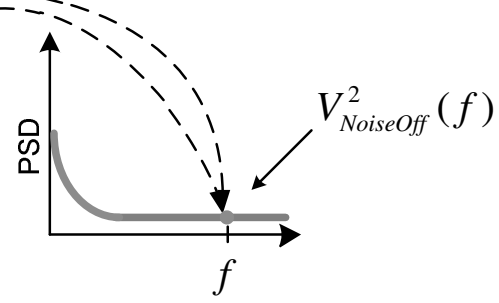

Offset Frequency $[\mathrm{Hz}]$

Figure 4: This diagram graphically represents the four measurement steps involved in calibrating the PM/AM noise standard. In (a) and (b), the down-converted signal power at ' $f$ ' is proportional to the noise standard carrier power at $v_{0}$. 


\subsection{Measurement of the noise floor power spectral density}

The carrier power of the PM/AM noise standard is turned OFF and the noise is turned OFF. The frequency of the offset generator kept at $v_{0}$. Next, a noise floor measurement of the downconversion process is made. The FFT analyzer must NOT be auto-ranged, and the range setting should be identical to that used in Section 5.3. Keeping the auto-range at the same level ensures that the noise floor of the FFT analyzer is the same for measurements 5.3 and 5.4. The PSD at offset frequency $f$ is measured in units of $V_{r m s}^{2} / H z$, utilizing a Hanning window. The PSD of the noise floor at $f$ will contain noise contributions from the down-converter components, such as the mixer, IF amplifier, the noise of the offset generator, and the input noise of the FFT spectrum analyzer. This measurement is graphically shown in Figure 4(d).

The measurement procedure described above is repeated at least three times for each carrier frequency. All measurements are made with a single one-time connection to the measurement system, and the same analyzer is used for all measurements. The absolute gain or frequency response of the down-converter and the spectrum analyzer are unimportant, because they are canceled due to the ratiometric relationship between the measurement of the carrier power and additive noise. This same characteristic also removes any inconsistency resulting from voltage standing wave ratio (VSWR) mismatch or connector interface non-repeatability.

The noise-voltage data are combined with the beat-power measurements described above according to the baseband measurement Eq. (18) to yield the relative power in either AM, 1/2 $S_{a}(f)$, or PM noise. 


\section{Measurement equation}

$\mathscr{L}(f)$ in Eq. (16) represents a ratio of power measurements performed at radio frequency (rf). A higher order correction, $K_{\beta}$, due to the small angle modulation approximation is introduced and Eq. (16) is rewritten as

$$
\mathscr{L}(f)=\frac{1}{2} S_{\alpha}(f)=\frac{1}{K_{\beta}} \frac{P S D\left[\text { Noise }\left(v_{0}-f\right)\right]+P S D\left[\text { Noise }\left(v_{0}+f\right)\right]}{4 \text { Carrier Power }} .
$$

The correction factor, $K_{\beta}$, is introduced only to propagate the error due to the approximation in the measurement equation. A value of unity will be used.

The actual measurements described in the above section are made at baseband by use of a downconverter. The following equation is used to calculate the AM or PM noise generated from these baseband measurements:

$$
\mathscr{L}(f)=\frac{1}{2} S_{a}(f)=\frac{1}{4} \frac{1}{K_{N L} K_{R F} K_{B W} K_{\beta}}\left\{\frac{P S D\left[V_{\text {NoiseOn }}^{2}(f)\right]}{\frac{V_{\text {Beat }-}^{2}(f)+V_{\text {Beat }+}^{2}(f)}{2}}\right\} .
$$

$V_{\text {NoiseOn }}^{2}(f)$ is the additive noise down-converted from both the upper and lower $\left(v_{0} \pm f\right)$ sidebands to baseband. $V_{\text {Beat }}^{2}(f)$ is the carrier power down-converted via either the upper or lower sideband to a baseband beat of $f . K_{N L}, K_{R F}$, and $K_{B W}$ are correction factors needed to account for nonlinearity, frequency response, and bandwidth errors in the baseband measurement. Appendix A shows the equivalence of Eqs. (17) and (18), and Appendix B describes the techniques to measure these correction factors.

When the bias due to background measurement noise is removed, Eq. (18) can be written as follows:

$$
\mathscr{L}(f)=\frac{1}{2 K_{N L} K_{R F} K_{B W} K_{\beta}} \frac{P S D\left[V_{\text {NoiseOn }, M}^{2}(f)\right]\left(1-\frac{P S D\left[V_{\text {NoiseOff, } M}^{2}(f)\right]}{P S D\left[V_{\text {NoiseOn }, M}^{2}(f)\right]}\right)}{\left(V_{\text {Beat }-, M}^{2}(f)+V_{\text {Beat }, M}^{2}(f)\right)\left(1-\frac{1}{S N R}\right)} .
$$

$V_{\text {Noiseoff }, M}^{2}(f)$ and SNR are respectively the noise floor of the measurement system for the additive noise measurement and the signal-to-noise power ratio of the carrier measurements. 
The same SNR is assumed for both $V_{\text {Beat }}$ and $V_{\text {Beat }}$ measurements. The logarithmic form of Eq. (19) is given by:

$$
\begin{aligned}
10 \log \mathscr{L}(f)=10 \log P S D\left[V_{\text {NoiseOn }, M}^{2}(f)\right]+10 \log \left(1-\frac{P S D\left[V_{\text {Noiseoff }, M}^{2}(f)\right]}{P S D\left[V_{\text {NoiseOn }, M}^{2}(f)\right]}\right) \\
\quad-10 \log \left(V_{\text {Beat }, M}^{2}(f)+V_{\text {Beat }, M}^{2}(f)\right)-10 \log \left(1-\frac{1}{S N R}\right)-10 \log \left(2 K_{N L} K_{R F} K_{B W} K_{\beta}\right) .
\end{aligned}
$$

The first term is the measured noise level with noise source $\mathrm{ON}$, the second term is the bias on the measured noise due to the noise floor (measured with the noise source OFF), the third term is the average of the measured carrier power down-converted via both the upper and lower sidebands, the fourth term is the bias on the carrier power measurements due to the background noise, and the last term contains the correction factors. 


\section{Estimation of uncertainty}

Normalizing the PSD to a $1 \mathrm{~Hz}$ bandwidth and rewriting the measurement Eq. (19) gives

$$
\mathscr{L}(f)=\frac{1}{2 K_{N L} K_{R F} K_{B W} K_{\beta}} \frac{V_{\text {NoiseOn }, M}^{2}(f)-V_{\text {NoiseOff }, M}^{2}(f)}{\left(V_{\text {Beat }-, M}^{2}(f)+V_{\text {Beat }, M}^{2}(f)\right)\left(1-\frac{1}{S N R}\right)}
$$

From the Guidelines for Evaluating and Expressing the Uncertainty of NIST Measurement Results [15][16], the equation for the combined uncorrelated uncertainties $\left(u_{C}\right)$ for a measurement result, $y$, is written as

$$
\begin{aligned}
& y=f\left(x_{1}, x_{2}, \ldots, x_{N}\right) \\
& u_{C}^{2}(y)=\sum_{i=1}^{N} \underbrace{\left(\frac{\partial f}{\partial x_{i}}\right)^{2}}_{\substack{\text { Sensitivity } \\
\text { Coefficients }}} u^{2}\left(x_{i}\right) .
\end{aligned}
$$

In terms of fractional uncertainties Eq. (22) is

$$
\begin{aligned}
& \overbrace{\frac{u_{C}^{2}(y)}{y^{2}}}^{\substack{\text { Total } \\
\text { Uractional } \\
\text { Uncertainty }}}=\sum_{i=1}^{N} \overbrace{\left(\frac{\partial f}{\partial x_{i}}\right)^{2} \frac{x_{i}^{2}}{y^{2}}}^{\text {Multiplier }} \overbrace{\frac{u^{2}\left(x_{i}\right)}{x_{i}^{2}}}^{\begin{array}{c}
\text { ith Component } \\
\text { Fractional } \\
\text { Uncertainty }
\end{array}} \\
& \sigma_{C}^{2}=\sum_{i=1}^{N} \overbrace{\left(\frac{\partial f}{\partial x_{i}}\right)^{2} \frac{x_{i}^{2}}{y^{2}} \overbrace{\sigma_{i}^{2}}^{\text {Multiplier }} \text {. }}^{\begin{array}{c}
\text { ith Component } \\
\text { Fractional }
\end{array}} \text {. }
\end{aligned}
$$

$\sigma^{2}$ is used here to represent the fractional variance. The combined uncertainty for $\mathscr{L}(f)$ is therefore

$$
u_{C}^{2}(\mathscr{L})=\left[\begin{array}{l}
\left(\frac{\partial \mathscr{L}}{\partial V_{\text {NoiseOn.M }}}\right)^{2} u^{2}\left(V_{\text {NoiseO }, M}\right)+\left(\frac{\partial \mathscr{L}}{\partial V_{\text {NoiseOff }}}\right)^{2} u^{2}\left(V_{\text {Noise Off }, M}\right) \\
\left.+\left(\frac{\partial \mathscr{L}}{\partial K_{N L}}\right)^{2} u^{2}\left(K_{N L}\right)+\left(\frac{\partial \mathscr{L}}{\partial K_{R F}}\right)^{2} u^{2}\left(K_{R F}\right)+\left(\frac{\partial \mathscr{L}}{\partial K_{B W}}\right)^{2} u^{2}\left(K_{B W}\right)+\left(\frac{\partial \mathscr{L}}{\partial K_{\beta}}\right)^{2} u^{2}\left(K_{\beta}\right)\right] \\
+\left(\frac{\partial \mathscr{L}}{\partial V_{\text {Beat }, M}}\right)^{2} u^{2}\left(V_{\text {Beat }, M}\right)+\left(\frac{\partial \mathscr{L}}{\partial V_{\text {Beat }, M}}\right)^{2} u^{2}\left(V_{\text {Beat }, M}\right)+\left(\frac{\partial \mathscr{L}}{\partial S N R}\right)^{2} u^{2}(S N R)
\end{array}\right]
$$


The individual multipliers of (23) are

$$
\begin{aligned}
& \left(\frac{\partial \mathscr{L}}{\partial V_{\text {NoiseOn }, M}}\right)^{2} \frac{V_{\text {NoiseOn, }, M}^{2}}{\mathscr{L}^{2}}=\frac{4 V_{\text {NoiseOn }, M}^{4}}{\left(V_{\text {NoiseOn }, M}^{2}-V_{\text {NoiseOff }, M}^{2}\right)^{2}} \\
& \left(\frac{\partial \mathscr{L}}{\partial V_{\text {NoiseOff }, M}}\right)^{2} \frac{V_{\text {NoiseOff }, M}^{2}}{\mathscr{L}^{2}}=\frac{4 V_{\text {NoiseOff }, M}^{4}}{\left(V_{\text {NoiseOn }, M}^{2}-V_{\text {NoiseOff }, M}^{2}\right)^{2}} \\
& \left(\frac{\partial \mathscr{L}}{\partial K_{N L}}\right)^{2} \frac{K_{N L}^{2}}{\mathscr{L}^{2}}=\left(\frac{\partial \mathscr{L}}{\partial K_{R F}}\right)^{2} \frac{K_{R F}^{2}}{\mathscr{L}^{2}}=\left(\frac{\partial \mathscr{L}}{\partial K_{B W}}\right)^{2} \frac{K_{B W}^{2}}{\mathscr{L}^{2}}=\left(\frac{\partial \mathscr{L}}{\partial K_{\beta}}\right)^{2} \frac{K_{\beta}^{2}}{\mathscr{L}^{2}}=1 \\
& \left(\frac{\partial \mathscr{L}}{\partial V_{\text {Beat }-, M}}\right)^{2} \frac{V_{\text {Beat }-, M}^{2}}{\mathscr{L}^{2}}=\frac{4 V_{\text {Beat }, M}^{4}}{\left(V_{\text {Beat }-, M}^{2}+V_{\text {Beat }, M}^{2}\right)^{2}} \\
& \left(\frac{\partial \mathscr{L}}{\partial V_{\text {Beat }+, M}}\right)^{2} \frac{V_{\text {Beat }+, M}^{2}}{\mathscr{L}^{2}}=\frac{4 V_{\text {Beat }+, M}^{4}}{\left(V_{\text {Beat }-, M}^{2}+V_{\text {Beat }+, M}^{2}\right)^{2}} \\
& \left(\frac{\partial \mathscr{L}}{\partial S N R}\right)^{2} \frac{S N R^{2}}{\mathscr{L}^{2}}=\frac{1}{(S N R-1)^{2}}
\end{aligned}
$$

The multipliers are simplified by making the following assumptions:

$$
\begin{aligned}
& V_{\text {NoiseOn }, M} \gg V_{\text {NoiseOff }, M} \\
& V_{\text {Beat }+, M} \cong V_{\text {Beat }-, M} \\
& \text { SNR }>1 .
\end{aligned}
$$

The combined fractional uncertainty, $\sigma_{C}$, obtained by combining the individual fractional uncertainties, whether arising from Type A evaluation or Type B evaluation [15], is

$$
\sigma_{C}^{2}=\frac{4 \sigma_{\text {NoiseOn }}^{2}+\sigma_{\text {Beat }-}^{2}+\sigma_{B e a t+}^{2}}{n_{\text {set }}}+\sigma_{N L}^{2}+\sigma_{R F}^{2}+\sigma_{B W}^{2}+\sigma_{\beta}^{2}+\sigma_{L R}^{2},
$$

where $n_{\text {set }}$ is the number of repeated measurement sets in a given calibration. $\sigma_{L R}$ is the uncertainty characterizing the long-term reproducibility of the PM/AM noise standard between calibration cycles. This includes the combined effect of temperature variations, connector mismatch, long-term variations of noise or carrier power, effects due to shock during shipping, and other unknown effects. $\sigma_{\text {NoiseOn }}$ and $\sigma_{\text {Beat }}$ include uncertainties due to the average of a random process given by 


$$
\begin{aligned}
& \sigma_{\text {NoiseOn }}^{2}=\sigma_{N-F F T A v e}^{2}+\sigma_{S R}^{2}=\left(\frac{1}{\sqrt{N_{\text {Noise }}}}\right)^{2}+\sigma_{S R}^{2}, \\
& \sigma_{\text {Beat }}^{2}=\sigma_{B-F F T A v e}^{2}+\sigma_{S R}^{2}=\left(\sqrt{\frac{2}{N_{\text {Beat }}}} \frac{1}{S N R}\right)^{2}+\sigma_{S R}^{2} .
\end{aligned}
$$

$N_{N o i s e}$ and $N_{\text {Beat }}$ are the number of FFT averages used for the noise and beat measurements respectively. $\sigma_{S R}$ is the short-term repeatability of the PM/AM noise standard during a single calibration. This includes the variation due to temperature fluctuations and variability in the insertion loss of the internal relays used to enable and disable the carrier and noise source for the calibration. Appendix C presents a derivation of (32) describing the error of signal power measurements in the presence of background noise.

The expanded uncertainty (U) is equal to $k \sigma_{c}$, where $k$ is the coverage factor and is chosen to be 2 for a $95.45 \%$ level of confidence [15]. 


\section{Uncertainty budget}

As stated in the Introduction, the AM and PM noise of the noise standard are usually measured two times at NIST, once before sending it ("out" measurement) to the customer and a second time after receiving it back ("in" measurement). The combined uncertainty includes the contributions of different estimated errors associated with the noise-standard calibration. In practice, all estimated errors are expressed in the same domain [linear (\%) or $\log (\mathrm{dB})]$ for the calculation of combined uncertainty. Typically, the final measurement results for PM and AM noise are reported in the log domain $(\mathrm{dBc} / \mathrm{Hz})$. However, in this calibration, not all errors are available in the same domain. For example, the uncertainty due to a FFT average is statistically calculated in the linear domain, whereas the uncertainty in the linearity of the FFT analyzer is provided by the manufacturer in $\mathrm{dB}$. Other errors associated with the noise standard and/or calibration that are experimentally measured can be calculated either in $\mathrm{dB}$ or $\%$. We chose to write the measurement and uncertainty equations in the linear domain and convert any errors expressed in $\mathrm{dB}$ to the linear domain. When converting $\mathrm{dB}$ uncertainties to linear, the upper and lower confidence range can be unequal for larger errors. In this document, we will convert all the $\mathrm{dB}$ errors to their linear values and consider the largest value if they are unequal for the uncertainty calculation. Certain assumptions concerning distributions in $\mathrm{dB}$ cannot always be properly converted to linear, and vice-versa.

The combined uncertainty is calculated entirely in terms of linear fractional uncertainty and the final expanded uncertainty (U) result is converted to $\mathrm{dB}$. The uncertainty budget for the calibration of the PM/AM noise standard is given in Table 1.

Table 1- Uncertainty budget: PM/AM noise measurement of the noise standard

\begin{tabular}{|c|c|c|c|c|c|c|c|}
\hline Sources of Error & $\begin{array}{l}\text { Estimated } \\
\text { Fractional } \\
\text { Error, } \%\end{array}$ & Effect & Type & Distribution & Divisor & $\begin{array}{c}\text { Standard } \\
\text { Fractional } \\
\text { Uncertainty } \\
(\sigma), \%\end{array}$ & Symbol \\
\hline $\begin{array}{l}\text { Number of FFT averages } \\
\text { for noise measurement } \\
N_{\text {Noise }}=10,000\end{array}$ & 1.0 & Random & A & Normal & 1 & 1.0 & $\sigma_{N-F F T A v e}$ \\
\hline $\begin{array}{l}\text { Number of FFT averages } \\
\text { for beat measurement } \\
N_{\text {Beat }}=100 \\
S N R=1000\end{array}$ & 0.0 & Random & A & Normal & 1 & 0.0 & $\sigma_{B \text {-FFTAve }}$ \\
\hline $\begin{array}{l}\text { Uncertainty in the } \\
\text { estimated measurement } \\
\text { bandwidth for the PSD } \\
\text { function. }(0.05 \mathrm{~dB})\end{array}$ & 1.2 & Systematic & B & Rectangular & $\sqrt{3}$ & 0.7 & $\sigma_{B W}$ \\
\hline $\begin{array}{l}\text { Frequency response of the } \\
\text { down convertor (includes } \\
\text { offset signal generator } \\
\text { and mixer) at rf }\end{array}$ & 2.3 & Systematic & B & Rectangular & $\sqrt{3}$ & 1.3 & $\sigma_{R F}$ \\
\hline $\begin{array}{l}\text { FFT and mixer linearity at } \\
\text { baseband }\end{array}$ & 4.7 & Systematic & B & Rectangular & $\sqrt{3}$ & 2.7 & $\sigma_{N L}$ \\
\hline $\begin{array}{l}\text { Small angle modulation } \\
\text { approximation. } \beta=0.02\end{array}$ & 0.0 & Systematic & B & Fixed value & 1 & 0.0 & $\overline{\sigma_{\beta}}$ \\
\hline $\begin{array}{l}\text { Short term noise standard } \\
\text { repeatability }\end{array}$ & 2.3 & Random & A & Normal & 1 & 2.3 & $\sigma_{S R}$ \\
\hline $\begin{array}{l}\text { Long term noise standard } \\
\text { reproducibility }\end{array}$ & 7.2 & Systematic & B & Rectangular & $\sqrt{3}$ & 4.1 & $\sigma_{L R}$ \\
\hline
\end{tabular}


By use of Table 1 and Eq. (32) the expanded fractional uncertainty for values shown in this example is $\pm 11.5 \%$ or $\pm 0.5 \mathrm{~dB}$ for $n_{\text {set }}$ equal to 6 .

It should be noted that this calibration standard creates equal amount of AM and PM noise. When this type of standard is used to evaluate AM/PM measurement systems, the AM-to-PM or PM-to-AM conversions in the detector cannot be ignored [17]. In a typical PM noise measurement, AM-to-PM conversion can typically be $15 \mathrm{~dB}$ or worse leading to an error of 0.3 $\%(0.1 \mathrm{~dB})$. 


\section{Validation of the measurement}

To further validate the calibration method, we use two different approaches. In the first approach, the noise of the standard is measured with different PM noise measurement systems and with different measurement techniques, for example, a direct-digital phase noise measurement system (DDPNMS) [18], or a photonic delay-line measurement system (PDLMS) [19]. The agreement between the results of the DDPNMS and/or PDLMS and the calibrated result validate the calibration method. The second approach is to calibrate a phase noise measurement system by use of a calibrated noise standard and compare the results with that obtained by other calibration methods such as a single-sideband modulation, PM/AM modulation, beat frequency, or static phase shift [7], [20]. All of these methods should give identical results within the measurement uncertainty.

\section{Acknowledgement}

The authors thank Fred Walls for useful discussions and thoughtful comments on this manuscript, and Tom Parker, Mike Lombardi, David Smith, and Danielle Lirette for their helpful suggestions in the preparation of this manuscript. 


\section{Appendix A. Verification of the baseband measurement equation}

The phase noise, $\mathscr{L}(f)$ and amplitude noise, $S_{\alpha}(f)$ generated by the PM/AM noise standard is given by

$$
\mathscr{L}(f)=\frac{1}{2} S_{a}(f)=\frac{1}{4 K_{\beta}} \frac{P S D\left[\text { Noise }\left(v_{0}-f\right)\right]+P S D\left[\text { Noise }\left(v_{0}+f\right)\right]}{\text { Carrier Power }} . \quad[\mathrm{dBc} / \mathrm{Hz} \text { or } 1 / \mathrm{Hz}]
$$

The noise represented by Eq. (A1) is not measured directly at rf, but rather indirectly via downconversion to baseband. The following equation, called the "measurement equation," describes the measurement at baseband:

$$
\mathscr{L}(f)=\frac{1}{4} \frac{1}{K_{N L} K_{R F} K_{B W} K_{\beta}} \frac{\left[\frac{V_{\text {NoiseOn }}^{2}(f)}{B W_{E s t}}\right]}{V_{\text {BeatAve }}^{2}(f)} .
$$

A proof follows that shows that the baseband measurement Eq. (A2) is equivalent to the definition (A1) when appropriate approximations and correction terms are defined. $V_{\text {Noise } n}^{2}(f)$ is the measured additive noise down-converted from both the upper and lower $\left(v_{0} \pm f\right)$ sidebands to baseband. $V_{\text {BeatAve }}^{2}(f)$ is the average of the measured carrier power down-converted via both the upper and lower sideband to a baseband frequency of $f . \quad B W_{E s t}$ is an estimate of the measurement bandwidth used to calculate the PSD function. $K_{N L}, K_{R F}, K_{B W}$ and $K_{\beta}$, to be defined later, are correction factors to account for nonlinearity, frequency response, bandwidth and small angle approximation errors in the baseband measurement.

The simplified function of the heterodyne down-converter is described as follows. An offset signal generator is connected to the LO port of a double-balanced mixer, and the PM/AM noise standard is connected to the RF port of the mixer via a large attenuator, ensuring linear conversion from the RF port to baseband. The gain of the down-converter for a given LO frequency $\left(v_{L O}\right)$ and RF frequency $\left(v_{R F}\right)$ to a baseband IF frequency $\left(f=\left|v_{L O}-v_{R F}\right|\right)$ is

$$
G\left(v_{L O}, v_{R F}, \text { level }\right)=g_{V S W R} g_{I F}\left(\left|v_{L O}-v_{R F}\right|\right) g_{R F}\left(v_{L O}, v_{R F}\right) g_{L V L}(P)
$$

where, $g$ 's are different gain terms. $g_{V S W R}$ represents any mismatch error due to VSWR [21], $g_{I F}(f)$ represents the baseband frequency response at IF, $g_{R F}\left(v_{L O}, v_{R F}\right)$ represents the $\mathrm{rf}$ frequency response of the down-converter at its two operating frequencies, and the $g_{L V L}(P)$ represents the response of the down-converter at a specific power, $P$, at the RF port. 
When the signals from the offset generator and the noise standard are mixed, two sidebands at $v_{0} \pm f$ are translated to a baseband frequency at $f$. The folded double-sideband downconversion is represented by Eq. (A4) and is shown graphically in Figure 5 [11].

$$
V_{\text {Baseband }}^{2}(f)=G^{2}\left(v_{0}, v_{0}-f, P\right) V_{P N S T}^{2}\left(v_{0}-f\right)+G^{2}\left(v_{0}, v_{0}+f, P\right) V_{P N S T}^{2}\left(v_{0}+f\right),
$$

where $V_{P N S T}$ is the signal voltage of the PM/AM noise standard at rf.

(a)

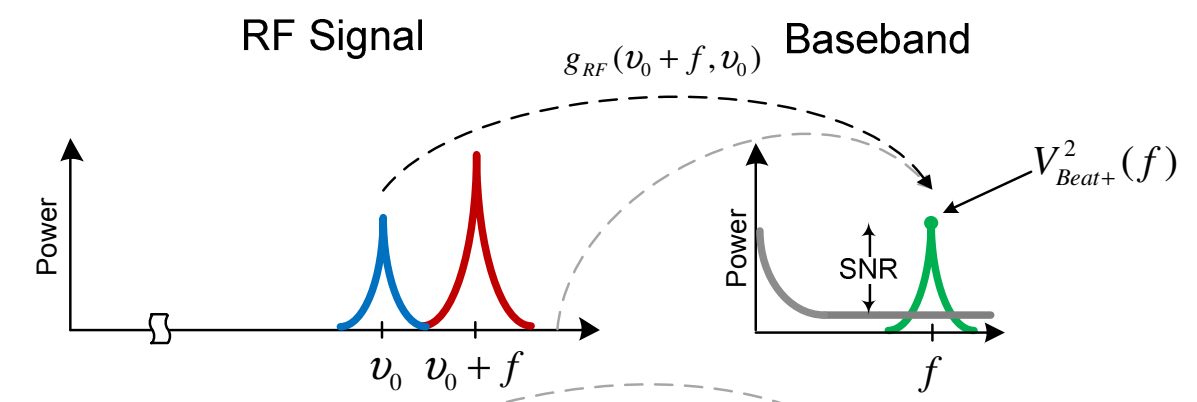

(b)

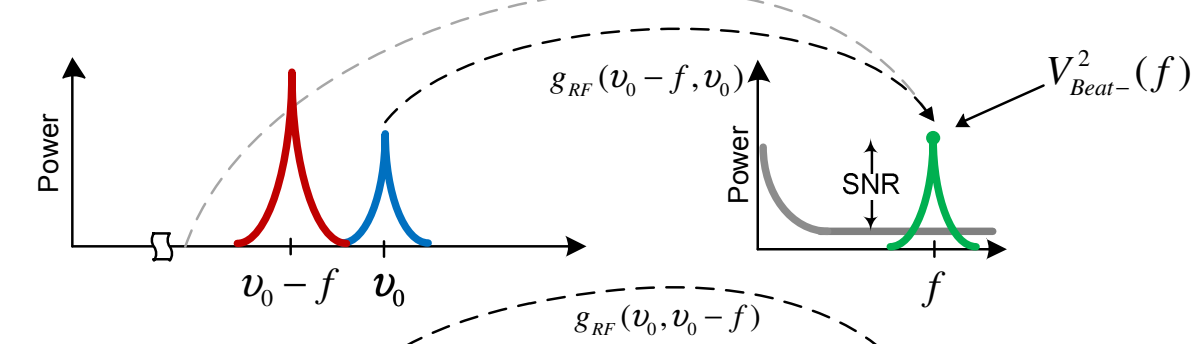

(c)

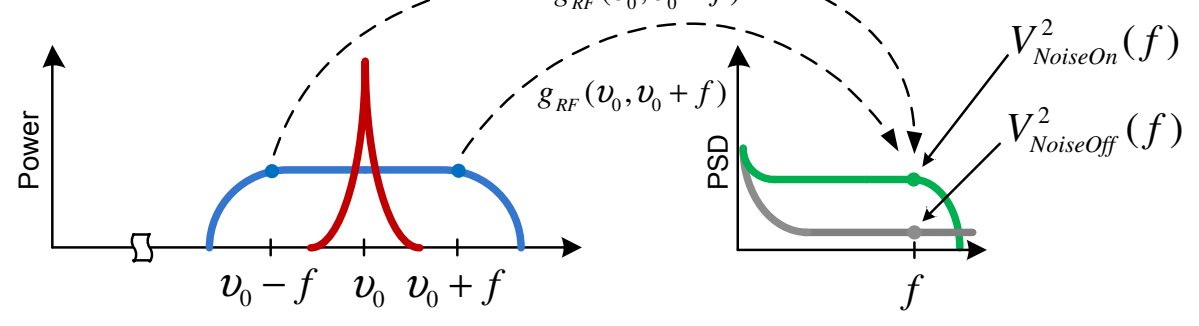

Frequency $[\mathrm{Hz}]$

Offset Frequency [Hz]

Figure 5. Graphical representation of the baseband calibration measurements. The red and blue traces represent the offset generator and the PM/AM noise standard, respectively. The green spectrum is the down-converted baseband signal. The dark gray trace is the noise floor of the measurement system. The dotted lines indicate the down-conversion gains, where the light gray dotted line indicates the undesired, negligible and hence ignored, down-converted image.

By use of Eq. (A4) the numerator of Eq. (A2) can be written as

$$
V_{\text {NoiseOn }}^{2}(f)=G^{2}\left(v_{0}, v_{0}-f, P_{\text {noise }}\right) V_{P N S T \text { (NoiseOn })}^{2}\left(v_{0}-f\right)+G^{2}\left(v_{0}, v_{0}+f, P_{\text {noise }}\right) V_{P N S T(\text { NoiseO } n)}^{2}\left(v_{0}+f\right) .
$$

$V_{P N S T \text { (NoiseOn) }}$ represents the signal voltage of the standard when the additive noise is enabled and the carrier is off. $P_{\text {noise }}$ is the power level at the RF port of the mixer during the noise 
measurement. The upper and lower sideband gains are factored out into a single term and simplified by a first order Taylor expansion when both sidebands are approximately equal as follows:

$$
\begin{aligned}
& c(x+y)=a x+b y \\
& c=\frac{a x+b y}{x+y} \\
& c=\frac{a+b}{2}+\mathrm{O} \text { when } x \cong y,
\end{aligned}
$$

where $\mathrm{O}$ contains the higher-order terms of the Taylor expansion. Therefore,

$$
V_{\text {NoiseOn }}^{2}(f)=\left[\frac{G^{2}\left(v_{0}, v_{0}-f, P_{\text {noise }}\right)+G^{2}\left(v_{0}, v_{0}+f, P_{\text {noise }}\right)}{2}+O\right]\left[V_{P N S T(\text { NoiseOn })}^{2}\left(v_{0}-f\right)+V_{P N S T(\text { NoiseOn })}^{2}\left(v_{0}+f\right)\right]
$$

Similarly, $V_{\text {BeatAve }}^{2}(f)$ in the denominator of (A2) can be rewritten, when the contribution from the unwanted and negligible down-converted image is ignored, as

$$
V_{\text {BeatAve }}^{2}(f)=\frac{G^{2}\left(v_{0}-f, v_{0}, P_{\text {carrier }}\right)+G^{2}\left(v_{0}+f, v_{0}, P_{\text {carrier }}\right)}{2} V_{P N S T(\text { CarrierOn })}^{2}\left(v_{0}\right),
$$

where $V_{P N S T(\text { CarrierOn) }}$ represents the signal voltage of the standard when the additive noise is off and the carrier is enabled, and $P_{\text {carrier }}$ represents the power level at the RF port of the mixer during measurement of carrier power. Substituting the numerator and denominator in Eq. (A2) with Eq. (A7) and Eq. (A8) gives

$$
\mathscr{L}(f)=\frac{1}{4} \frac{K}{K_{N L} K_{R F} K_{B W} K_{\beta}} \frac{\left[\frac{V_{P N S T(\text { NoiseOn })}^{2}\left(v_{0}-f\right)+V_{P N S T(\text { NoiseOn })}^{2}\left(v_{0}+f\right)}{B W_{\text {Est }}}\right]}{V_{P N S T \text { NoiseOn })}^{2}\left(v_{0}\right)} .
$$

All common and similar terms are collected in the variable $K$, given by

$$
K=\frac{g_{V S W W}^{2} g_{y}^{2}(f)}{g_{V \text { SWR }}^{2} g_{I F}^{2}(f)} \frac{g_{L V L}^{2}\left(P_{\text {noise }}\right)}{g_{L V L}^{2}\left(P_{\text {carrier }}\right)} \frac{\left[g_{R F}^{2}\left(v_{0}, v_{0}-f\right)+g_{R F}^{2}\left(v_{0}, v_{0}+f\right)+2 O\right]}{\left[g_{R F}^{2}\left(v_{0}-f, v_{0}\right)+g_{R F}^{2}\left(v_{0}+f, v_{0}\right)\right]} \text {. }
$$

This clearly shows that the baseband gains as well as VSWR mismatches cancel for the measurement Eq. (A2).

Finally, the four correction factors $K_{N L}, K_{R F}, K_{B W}$ and $K_{\beta}$ are defined. $K_{N L}$ defines the change in gain that occurs due to nonlinearity in the down-converter and FFT analyzer due to a difference in power level between noise and carrier power measurements: 


$$
K_{N L}=\left(\frac{g_{L V L}\left(P_{\text {noise }}\right)}{g_{L V L}\left(P_{\text {carrier }}\right)}\right)^{2} .
$$

$K_{R F}$ is the ratio of the effective power gain between noise and carrier power measurements. Ideally, this number should be unity; however, it is affected by the non-flat amplitude response of the offset generator. It also contains the asymmetrical frequency response of the mixer at its LO and RF ports as well as the higher-order terms of the Taylor expansion of example (A6):

$$
K_{R F}=\frac{g_{R F}^{2}\left(v_{0}, v_{0}-f\right)+g_{R F}^{2}\left(v_{0}, v_{0}+f\right)+2 O}{g_{R F}^{2}\left(v_{0}-f, v_{0}\right)+g_{R F}^{2}\left(v_{0}+f, v_{0}\right)} .
$$

$K_{B W}$ is a correction for an imperfect power spectral density (PSD) function given by

$$
K_{B W}=\frac{B W_{A c t u a l}}{B W_{E s t}},
$$

where $B W_{\text {Actual }}$ is the true measurement bandwidth.

Using Eqs. (A11), (A12) and (A13) in Eq. (A9), we see that $\mathscr{L}(f)$ at the baseband measurement equation is equal to the original definition of $\mathscr{L}(f)$ at $\mathrm{rf}(\mathrm{A} 1)$ as follows:

$$
\begin{aligned}
\mathscr{L}(f) & =\frac{1}{4 K_{\beta}} \frac{\left[\frac{V_{P N S T(\text { NoiseOn })}^{2}\left(v_{0}-f\right)+V_{P N S T(\text { NoiseOn })}^{2}\left(v_{0}+f\right)}{B W_{\text {Actual }}}\right]}{V_{P N S T(\text { CarrierOn })}^{2}\left(v_{0}\right)} \\
& =\frac{1}{4 K_{\beta}} \frac{P S D\left[V_{P N S T(\text { NoiseOn })}^{2}\left(v_{0}-f\right)+V_{P N S T \text { NoiseOn })}^{2}\left(v_{0}+f\right)\right]}{V_{P N S T(\text { CarrierOn })}^{2}\left(v_{0}\right)} .
\end{aligned}
$$




\section{Appendix B. Measurement of correction factors, $\boldsymbol{K}_{N L}, \boldsymbol{K}_{R F}, \boldsymbol{K}_{B W}$ and $\boldsymbol{K}_{\beta}$}

(i) Set-up for measuring nonlinearity of the down-converter and the FFT analyzer $K_{N L}$

As discussed earlier, $K_{N L}$ is defined as the change in gain that occurs due to nonlinearity in the down-converter and FFT analyzer due to a difference in power level between noise and carrier power measurements and given by Eq. (A11). The carrier power is measured with additive noise off, while the noise-power measurements are made with the carrier off.

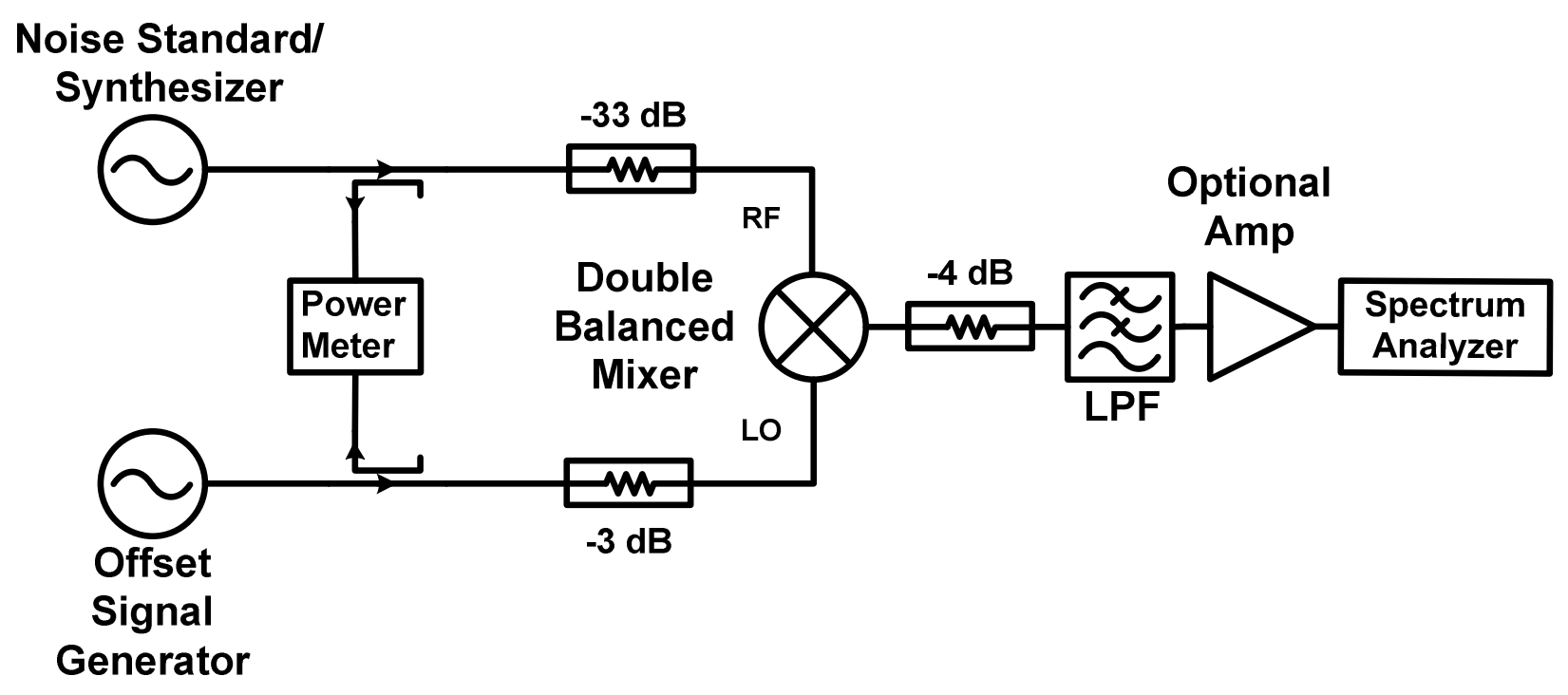

Figure 6. Measurement setup for the estimation of $K_{N L}$ and $K_{R F}$.

The measurement system used to measure uncertainty due to the FFT and the mixer nonlinearity at baseband is shown in Figure 6. The frequency of the offset signal generator is set at $v_{0}+f$ and the noise standard at $v_{0}$. A portion of the signal from the noise standard is coupled out and connected to a NIST-calibrated power meter with very low noise floor $(-60 \mathrm{dBm})$. First, the carrier power $\left(P_{\text {carrier }}\right)$ and noise power $\left(P_{\text {noise }}\right)$ of the standard are measured with the power meter. The noise standard is then replaced by a rf synthesizer at frequency $v_{0}$ and power equal to $P_{\text {carrier }}$. The corresponding down-converted power $\left(P_{C \text {-Beat }}\right)$ is measured on the FFT analyzer by use of a flattop window. Next, the synthesizer power is adjusted to equal the integrated noise power of the noise standard $\left(P_{\text {noise }}\right)$, and the down-converted power $\left(P_{N \text {-Beat }}\right)$ is measured on the FFT analyzer. From these measurements $K_{N L}$ can be calculated as:

$$
K_{N L}=\left(\frac{g_{L V L}\left(P_{\text {noise }}\right)}{g_{L V L}\left(P_{\text {carrier }}\right)}\right)^{2}=\frac{P_{N-\text { Beat }}}{P_{\text {noise }}} / \frac{P_{C-\text { Beat }}}{P_{\text {carrier }}} .
$$


These measurements are repeated for all the specified offset frequencies $( \pm f)$ at each of the three carrier frequencies. Because the mixer is operating in linear mode, $K_{N L}$ should ideally be equal to 1 . The maximum observed error from unity, $\delta_{N L}$, can be used to construct a rectangular distribution for $K_{N L}$. Therefore,

$$
K_{N L}=1 \pm \frac{\delta_{N L}}{\sqrt{3}}=1 \pm \sigma_{N L}
$$

where $\sigma_{N L}$ is the fractional uncertainty of $K_{N L}$.

\section{(ii) Set-up for measuring frequency response of the down convertor at $\mathrm{rf}-K_{R F}$}

$K_{R F}$ is the ratio of the effective power gain between noise and carrier power measurements and given by Eq. (A12). The experimental set-up for determining $K_{R F}$ is the same as shown in Figure 6. In this case, the beat power, $P_{C \text {-Beat }}\left(v_{L O}, v_{R F}\right)$, is measured for four different LO and RF frequency combinations. For all four measurements, the power of the synthesizer is adjusted so that its power stays constant at $P_{\text {carrier. }} . K_{R F}$ can then be calculated from

$$
K_{R F}=\frac{P_{C-\text { Beat }}\left(v_{0}, v_{0}-f\right)+P_{C-\text { Beat }}\left(v_{0}, v_{0}+f\right)}{P_{C-\text { Beat }}\left(v_{0}-f, v_{0}\right)+P_{C-\text { Beat }}\left(v_{0}+f, v_{0}\right)} .
$$

These measurements are repeated for all the specified offset frequencies $( \pm f)$ at each of the three carrier frequencies. $K_{R F}$ should ideally be equal to 1 . The maximum observed error from unity, $\delta_{R F}$, can be used to construct a rectangular distribution for $K_{R F}$. Therefore,

$$
K_{R F}=1 \pm \frac{\delta_{R F}}{\sqrt{3}}=1 \pm \sigma_{R F}
$$

where $\sigma_{R F}$ is the fractional uncertainty of $K_{R F}$.

\section{(iii) Measurement of PSD correction factor $-K_{B W}$}

$K_{B W}$ as defined in Eq. (A13) is a correction for an imperfect power spectral density function. Since the FFT is digitally implemented, the resolution bandwidth of measurement and associated windows are well defined mathematically. Exact details of the FFT implementation depend on the manufacturer. The FFT manual should be checked for possible bandwidth uncertainty for a given instrument. Verification of the PSD function can also be implemented by use of a well characterized filter and a calibrated power meter [14]. In this case, we use $K_{B W}$ equal to unity with a fractional uncertainty, $\sigma_{B W}=0.7 \%$. 


\section{(iv) Calculation of small angle modulation correction factor $-K_{\beta}$}

$K_{\beta}$ is a correction to the small angle modulation approximation used to calculate $\mathscr{L}(f)$ from a single sideband power ratio. An effective peak phase modulation $\beta$ is obtained by integrating the phase noise and utilized to determine $K_{\beta}$ as follows

$$
\beta=\sqrt{2 \int_{f_{\mathrm{L}}}^{f_{\mathrm{U}}} \mathrm{S}_{\phi}(f) \mathrm{d} f} .
$$

Typically, $f_{\mathrm{U}}$ is the half-bandwidth of the band-pass filter in the noise standard.

$K_{\beta}$ is chosen to be unity with a fractional uncertainty $\sigma_{\beta}$.

$$
K_{\beta}=1 \pm\left(\varepsilon_{\beta}-1\right)=1 \pm\left(\frac{J_{1}^{2}(\beta) / J_{0}^{2}(\beta)}{\beta^{2} / 4}-1\right)=1 \pm \sigma_{\beta} .
$$




\section{Appendix C. Error of signal power measurements in the presence of background noise}

Consider the fast Fourier transform (FFT) of a signal of amplitude A and frequency $f$, such that the signal occurs in a single-frequency FFT bin (no leakage to neighboring bins) with white uncorrelated noise in that bin (and in every bin). Measurements are repeated $N$ times and averaged. Let the noise in the $i^{\text {th }}$ measurement be represented by $n_{i}$, a Gaussian sequence of values with zero mean and covariance $\sigma^{2}$. Then if the signal amplitude in the frequency bin of interest is $S_{i}$, the average of the measurements is

$$
M=\frac{1}{N} \sum_{i=1}^{N}\left(S_{i}+n_{i}\right)
$$

We can regard the expectation value of a limited set of measurement as the simple average:

$$
\langle M\rangle=\frac{1}{N} \sum_{i=1}^{N} S_{i}
$$

The expected rms error of the measurement due only to the noise is

$$
E=\sqrt{\left\langle(M-\langle M\rangle)^{2}\right\rangle}=\sqrt{\left\langle\left(\frac{1}{N} \sum_{i=1}^{N}\left(n_{i}\right)\right)^{2}\right\rangle}=\frac{1}{N} \sqrt{\left\langle\sum_{i, j=1}^{N}\left(n_{i} n_{j}\right)\right\rangle} .
$$

Given the Gaussian uncorrelated property of the noise, we can write

$$
\left\langle n_{i} n_{j}\right\rangle=\sigma^{2} \delta_{i j}
$$

which is zero unless $i=j$. There are $N$ such terms in the sum in Eq. (C3), so

$$
E=\frac{1}{N} \sqrt{N \sigma^{2}}=\frac{\sigma}{\sqrt{N}}
$$

Thus the measurement can be reported with a statistical error given by

$$
\frac{1}{N} \sum_{i=1}^{N} S_{i} \pm \frac{\sigma}{\sqrt{N}}
$$

Suppose, however, that the FFT is squared and then averaged. We then have for the measurement

$$
M=\frac{1}{N} \sum_{i=1}^{N}\left|S_{i}+n_{i}\right|^{2}=\frac{1}{N} \sum_{i=1}^{N}\left(\left|S_{i}\right|^{2}+\left(S_{i} n_{i}^{*}+S_{i}^{*} n_{i}\right)+\left|n_{i}\right|^{2}\right)
$$

The middle terms drop out, because there is no correlation between the signal and the noise. In the last sum, the average of the noise is $\sigma^{2}$,by Eq. (C4). The measurement is then 


$$
M=\frac{1}{N} \sum_{i=1}^{N}\left|S_{i}\right|^{2}+\sigma^{2}
$$

If the noise power is known, it can be subtracted off and the measurement of the average signal power reported as

$$
M-\sigma^{2}=\frac{1}{N} \sum_{i=1}^{N}\left|S_{i}\right|^{2}
$$

Apart from the propagation of error that occurs in this subtraction, there will be some error associated with the measurement itself. This can be denoted by $E$, where

$$
E^{2}=\left\langle(M-\langle M\rangle)^{2}\right\rangle \text {. }
$$

Writing this out,

$$
E^{2}=\left\langle\left(\frac{1}{N} \sum_{i=1}^{N}\left|S_{i}+n_{i}\right|^{2}-\left(\frac{1}{N} \sum_{i=1}^{N}\left|S_{i}\right|^{2}+\sigma^{2}\right)\right)^{2}\right\rangle
$$

There is a rather subtle change in the order in which the operations are performed. If we again assume no correlation between signal and noise, the terms involving the signal in the above equation all cancel out or vanish. Then

$$
E^{2}=\left\langle\left(\frac{1}{N} \sum_{i=1}^{N}\left|n_{i}\right|^{2}-\left(\sigma^{2}\right)\right)^{2}\right\rangle=\left\langle\left(\frac{1}{N^{2}} \sum_{i, j=1}^{N}\left|n_{i}\right|^{2}\left|n_{j}\right|^{2}-2 \frac{1}{N} \sum_{i=1}^{N}\left|n_{i}\right|^{2}\left(\sigma^{2}\right)+\sigma^{4}\right)\right\rangle \text {. }
$$

Evaluating each of these terms one by one, in the first sum there are $\mathrm{N}$ terms with $i=j$. These will contribute the amount

$$
\frac{1}{N}\left\langle n_{i}^{4}\right\rangle
$$

There are additional contributions in the first sum with $i \neq j$, where there are $N^{2}-N=N(N-1)$ terms, each contributing $\left(\sigma^{2}\right)^{2}$. The first sum therefore contributes a total of

$$
\left\langle\frac{1}{N^{2}} \sum_{i, j=1}^{N}\left|n_{i}\right|^{2}\left|n_{j}\right|^{2}\right\rangle=\frac{\left\langle\left|n_{i}\right|^{4}\right\rangle}{N}+\frac{N(N-1)}{N^{2}} \sigma^{4} .
$$

In the second term of Eq. $(\mathrm{C} 12)$, there are $N$ terms each contributing $\sigma^{4}$, so the second term contributes

$$
-\frac{2}{N} N \sigma^{4}=-2 \sigma^{4}
$$

The last term contributes

$$
+\sigma^{4}
$$

Collecting all the terms, the error is given by

$$
E^{2}=\frac{\left\langle n_{i}^{4}\right\rangle}{N}+\frac{N(N-1)}{N^{2}} \sigma^{4}-2 \sigma^{4}+\sigma^{4}=\frac{\left\langle n_{i}^{4}\right\rangle}{N}-\frac{1}{N} \sigma^{4} .
$$

Thus, we have to estimate the fourth moment. In a normalized Gaussian distribution that is a function of $x$, the probability of getting a value $x$ within the increment $d x$ is 


$$
P(x) d x=\frac{1}{\sqrt{2 \pi} \sigma} e^{-\frac{x^{2}}{2 \sigma^{2}}} d x .
$$

The fourth moment is

$$
\left\langle x^{4}\right\rangle=\int_{-\infty}^{\infty} \frac{x^{4}}{\sqrt{2 \pi} \sigma} e^{-\frac{x^{2}}{2 \sigma^{2}}} d x=3 \sigma^{4}
$$

Thus the error is

$$
\begin{aligned}
& E^{2}=\frac{3 \sigma^{4}}{N}-\frac{\sigma^{4}}{N}=\frac{2 \sigma^{4}}{N} ; \\
& E=\sqrt{2 / N} \sigma^{2}
\end{aligned}
$$

The measurement of signal power can then be reported with an uncertainty as:

$$
M-\sigma^{2} \pm \frac{\sqrt{2} \sigma^{2}}{\sqrt{N}} \text {. }
$$

Expressing Eq. (C21) in terms of SNR gives

$$
\begin{array}{r}
S N R=\frac{M}{\sigma^{2}} \\
M\left(1-\frac{1 \pm \sqrt{2 / N}}{S N R}\right) .
\end{array}
$$




\section{REFERENCES}

[1] "Calibration Services User Guide," http://ts.nist.gov/MeasurementServices/Calibrations/pm_am_noise.cfm\#pm_am. [Online]. Available: http://ts.nist.gov/MeasurementServices/Calibrations/pm_am_noise.cfm\#pm_am.

[2] F. L. Walls, "Secondary standard for PM and AM noise at 5, 10, and $100 \mathrm{MHz}$," IEEE Transactions on Instrumentation and Measurement, vol. 42, no. 2, pp. 136-143, Apr. 1993.

[3] F. L. Walls, "Calibration system for determining the accuracy of phase modulation and amplitude modulation noise measurement apparatus," U.S. Patent 5,172,06415-Dec-1992.

[4] F. G. Ascarrunz and F. L. Walls, "A standard for PM and AM noise at 10.6, 21.2 and 42.4 GHz," in Frequency Control Symposium, 1996. 50th., Proceedings of the 1996 IEEE International., 1996, pp. 852-853.

[5] IEEE, "IEEE Standard Definitions of Physical Quantities for Fundamental Frequency and Time Metrology — Random Instabilities," IEEE Std 1139-1999, 1999. [Online]. Available: ISBN 0-73811754-4. [Accessed: 06-Aug-2010].

[6] D. B. Sullivan, D. W. Allan, D. A. Howe, and F. L. Walls, "Characterization of Clocks and Oscillators," in NIST Technical Note 1337, 1990.

[7] F. L. Walls and E. Ferre-Pikal, "Measurement of Frequency, Phase noise and Amplitude noise," in Wiley Encyclopedia of Electrical and Electronics Engineering, 1st ed., vol. 12, 24 vols., WileyInterscience, 1999, pp. 459-473.

[8] E. Rubiola, Phase Noise and Frequency Stability in Oscillators. Cambridge University Press, 2010.

[9] S. I. Baskakov, Signals and circuits. Mir Publishers, 1990.

[10] B. P. Lathi, Modern Digital and Analog Communications Systems, International 2 Revised ed. Henry Holt \& Company, 1983.

[11] M. Abramowitz and I. A. Stegun, Handbook of Mathematical Functions: with Formulas, Graphs, and Mathematical Tables. Dover Publications, 1965.

[12] F. L. Walls, "Correlation between upper and lower sidebands," IEEE Transactions on Ultrasonics, Ferroelectrics and Frequency Control, vol. 47, no. 2, pp. 407-410, Mar. 2000.

[13] R. P. Scott, C. Langrock, and B. H. Kolner, "High-dynamic-range laser amplitude and phase noise measurement techniques," Selected Topics in Quantum Electronics, IEEE Journal of, vol. 7, no. 4, pp. 641-655, 2001.

[14] F. L. Walls, D. B. Percival, and W. R. Irelan, "Biases and variances of several FFT spectral estimators as a function of noise type and number of samples," in Frequency Control, 1989., Proceedings of the 43rd Annual Symposium on, 1989, pp. 336-341.

[15] B. N. Taylor and C. E. Kuyatt, "Guidelines for Evaluating and Expressingthe Uncertainty of NIST Measurement Results," NIST Technical Note 1297, 1994.

[16] BIPM, "Evaluation of measurement data - Guide to the expression of uncertainty in measurement." 2008.

[17] E. Rubiola and R. Boudot, "The effect of AM noise on correlation phase-noise measurements," IEEE Transactions on Ultrasonics, Ferroelectrics and Frequency Control, vol. 54, no. 5, pp. 926932, May 2007.

[18] J. Grove, J. Hein, J. Retta, P. Schweiger, W. Solbrig, and S. R. Stein, "Direct-digital phase-noise measurement," in Frequency Control Symposium and Exposition, 2004. Proceedings of the 2004 IEEE International, 2004, pp. 287-291.

[19] E. Rubiola, E. Salik, S. Huang, N. Yu, and L. Maleki, "Photonic-delay technique for phase-noise measurement of microwave oscillators," Journal of the Optical Society of America B, vol. 22, no. 5, pp. 987-997, May 2005.

[20] F. L. Walls, "Method and apparatus for wide band phase modulation," U.S. Patent 4,968,90806Nov-1990.

[21] Hewlett Packard, "Fundamentals of RF and Microwave Power Measurements," Application Note, no. 64-1, 1978. 


\section{Errata}

1. p. 15, Equation (24) should be changed to

$$
u_{C}^{2}(\mathscr{L})=\left[\begin{array}{l}
\left(\frac{\partial \mathscr{L}}{\partial V_{\text {NoiseOn }, M}^{2}}\right)^{2} u^{2}\left(V_{\text {NoiseO }, M}^{2}\right)+\left(\frac{\partial \mathscr{L}}{\partial V_{\text {NoiseOff }, M}^{2}}\right)^{2} u^{2}\left(V_{\text {NoiseOff }, M}^{2}\right) \\
+\left(\frac{\partial \mathscr{L}}{\partial K_{N L}}\right)^{2} u^{2}\left(K_{N L}\right)+\left(\frac{\partial \mathscr{L}}{\partial K_{R F}}\right)^{2} u^{2}\left(K_{R F}\right)+\left(\frac{\partial \mathscr{L}}{\partial K_{B W}}\right)^{2} u^{2}\left(K_{B W}\right)+\left(\frac{\partial \mathscr{L}}{\partial K_{\beta}}\right)^{2} u^{2}\left(K_{\beta}\right) \\
+\left(\frac{\partial \mathscr{L}}{\partial V_{\text {Beat }, M}^{2}}\right)^{2} u^{2}\left(V_{\text {Beat }, M}^{2}\right)+\left(\frac{\partial \mathscr{L}}{\partial V_{\text {Beat }, M}^{2}}\right)^{2} u^{2}\left(V_{\text {Beat }, M}^{2}\right)+\left(\frac{\partial \mathscr{L}}{\partial S N R}\right)^{2} u^{2}(S N R)
\end{array}\right] .
$$

2. p. 16, Equations (25), (26), (28), (29) and (32) should be changed to

$$
\begin{aligned}
& \left(\frac{\partial \mathscr{L}}{\partial V_{\text {NoiseOn }, M}^{2}}\right)^{2} \frac{V_{\text {NoiseOn, }, M}^{4}}{\mathscr{L}^{2}}=\frac{V_{\text {NoiseOn }, M}^{4}}{\left(V_{\text {NoiseOn }, M}^{2}-V_{\text {NoiseOff }, M}^{2}\right)^{2}} \\
& \left(\frac{\partial \mathscr{L}}{\partial V_{\text {NoiseOff }, M}^{2}}\right)^{2} \frac{V_{\text {NoiseOff }, M}^{4}}{\mathscr{L}^{2}}=\frac{V_{\text {NoiseOff }, M}^{4}}{\left(V_{\text {NoiseOn }, M}^{2}-V_{\text {NoiseOff }, M}^{2}\right)^{2}} \\
& \left(\frac{\partial \mathscr{L}}{\partial V_{\text {Beat }-, M}^{2}}\right)^{2} \frac{V_{\text {Beat }-, M}^{4}}{\mathscr{L}^{2}}=\frac{V_{\text {Beat }-, M}^{4}}{\left(V_{\text {Beat }-, M}^{2}+V_{\text {Beat }, M}^{2}\right)^{2}} \\
& \left(\frac{\partial \mathscr{L}}{\partial V_{\text {Beat }+, M}^{2}}\right)^{2} \frac{V_{\text {Beat }+, M}^{4}}{\mathscr{L}^{2}}=\frac{V_{\text {Beat }+, M}^{4}}{\left(V_{\text {Beat }-, M}^{2}+V_{\text {Beat }+, M}^{2}\right)^{2}} \\
& \sigma_{C}^{2}=\frac{4 \sigma_{\text {NoiseOn }}^{2}+\sigma_{\text {Beat }-}^{2}+\sigma_{\text {Beat }+}^{2}}{4 n_{\text {set }}}+\sigma_{N L}^{2}+\sigma_{R F}^{2}+\sigma_{B W}^{2}+\sigma_{\beta}^{2}+\sigma_{L R}^{2},
\end{aligned}
$$

Equations have been corrected to reflect that the FFT averaging occurs on $V^{2}$ and not $V$.

3. The expression for mean-square fractional amplitude fluctuations and mean-square phase fluctuations in Equations (2), (3), and (12) and in the text on p. 2 and p. 4 are incorrect and should be replaced with $\left\langle\alpha(f)^{2}\right\rangle$ and $\left\langle\Delta \phi_{r m s}(f)^{2}\right\rangle$, respectively. 




\section{NIST Technical Publications}

\section{Periodical}

Journal of Research of the National Institute of Standards and Technology-Reports NIST research and development in metrology and related fields of physical science, engineering, applied mathematics, statistics, biotechnology, and information technology. Papers cover a broad range of subjects, with major emphasis on measurement methodology and the basic technology underlying standardization. Also included from time to time are survey articles on topics closely related to the Institute's technical and scientific programs. Issued six times a year.

\section{Nonperiodicals}

Monographs-Major contributions to the technical literature on various subjects related to the Institute's scientific and technical activities.

Handbooks-Recommended codes of engineering and industrial practice (including safety codes) devel- oped in cooperation with interested industries, professional organizations, and regulatory bodies.

Special Publications-Include proceedings of conferences sponsored by NIST, NIST annual reports, and other special publications appropriate to this grouping such as wall charts, pocket cards, and bibliographies.

National Standard Reference Data Series-Provides quantitative data on the physical and chemical properties of materials, compiled from the world's literature and critically evaluated. Developed under a worldwide program coordinated by NIST under the authority of the National Standard Data Act (Public Law 90-396). NOTE: The Journal of Physical and Chemical Reference Data (JPCRD) is published bimonthly for NIST by the American Institute of Physics (AlP). Subscription orders and renewals are available from AIP, P.O. Box 503284, St. Louis, MO 63150-3284.

Building Science Series-Disseminates technical information developed at the Institute on building materials, components, systems, and whole structures. The series presents research results, test methods, and performance criteria related to the structural and environmental functions and the durability and safety characteristics of building elements and systems.

Technical Notes-Studies or reports which are complete in themselves but restrictive in their treatment of a subject. Analogous to monographs but not so comprehensive in scope or definitive in treatment of the subject area. Often serve as a vehicle for final reports of work performed at NIST under the sponsorship of other government agencies. Voluntary Product Standards-Developed under procedures published by the Department of Commerce in Part 10, Title 15, of the Code of Federal Regulations. The standards establish nationally recognized requirements for products, and provide all concerned interests with a basis for common understanding of the characteristics of the products. NIST administers this program in support of the efforts of private-sector standardizing organizations.

Order the following NIST publications-FIPS and NISTIRs-from the National Technical Information Service, Springfield, VA 22161.

Federal Information Processing Standards Publications (FIPS PUB)-Publications in this series collectively constitute the Federal Information Processing Standards Register. The Register serves as the official source of information in the Federal Government regarding standards issued by NIST pursuant to the Federal Property and Administrative Services Act of 1949 as amended, Public Law 89-306 (79 Stat. 1127), and as implemented by Executive Order 11717 (38 FR 12315, dated May 11,1973) and Part 6 of Title 15 CFR (Code of Federal Regulations).

NIST Interagency or Internal Reports (NISTIR)-The series includes interim or final reports on work performed by NIST for outside sponsors (both government and nongovernment). In general, initial distribution is handled by the sponsor; public distribution is handled by sales through the National Technical Information Service, Springfield, VA 22161, in hard copy, electronic media, or microfiche form. NISTIR's may also report results of NIST projects of transitory or limited interest, including those that will be published subsequently in more comprehensive form. 
U.S. Department of Commerce

National Institute of Standards and Technology

325 Broadway

Boulder, CO 80305-3337

Official Business

Penalty for Private Use $\$ 300$ 